\title{
Practical analytical solutions for benchmarking of 2-D and 3-D geodynamic Stokes problems with variable viscosity
}

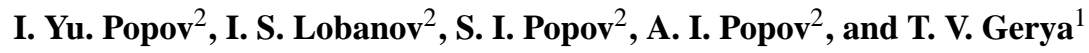 \\ ${ }^{1}$ Institute of Geophysics, Department of Earth Sciences, Swiss Federal Institute of Technology Zurich (ETH), \\ 5 Sonnegstrasse, 8092 Zurich, Switzerland \\ ${ }^{2}$ St. Petersburg National Research University of Information Technologies, Mechanics and Optics, 49 Kronverkskiy, \\ St. Petersburg, 197101, Russia
}

Correspondence to: I. Yu. Popov (popov1955@gmail.com)

Received: 20 November 2013 - Published in Solid Earth Discuss.: 9 December 2013

Revised: 15 April 2014 - Accepted: 22 April 2014 - Published: 10 June 2014

\begin{abstract}
Geodynamic modeling is often related with challenging computations involving solution of the Stokes and continuity equations under the condition of highly variable viscosity. Based on a new analytical approach we have developed particular analytical solutions for 2-D and 3-D incompressible Stokes flows with both linearly and exponentially variable viscosity. We demonstrate how these particular solutions can be converted into 2-D and 3-D test problems suitable for benchmarking numerical codes aimed at modeling various mantle convection and lithospheric dynamics problems. The Main advantage of this new generalized approach is that a large variety of benchmark solutions can be generated, including relatively complex cases with open model boundaries, non-vertical gravity and variable gradients of the viscosity and density fields, which are not parallel to the Cartesian axes. Examples of respective 2-D and 3-D MatLab codes are provided with this paper.
\end{abstract}

\section{Introduction}

Numerical modeling of geodynamic processes is recognized as a challenging computational problem which requires use of advanced computational techniques and development of powerful numerical tools (e.g., Ismail-Zadeh and Tackley, 2010, and references therein). One of the major challenges concerns solving of the inertia-free Stokes equation coupled to the incompressible continuity equation in a combination with strong viscosity variations in the computational domain. Consequently, benchmarking of numerical codes against analytical and numerical solutions constrained for various mechanical and thermomechanical Stokes flow problems is a common practice in computational geodynamics (e.g., Blankenbach et al., 1989; Moresi et al., 1996; van Keken et al., 2008; Gerya and Yuen, 2003, 2007; Deubelbeiss and Kaus, 2008; Duretz et al., 2011; Gerya et al., 2013; Popov, 2014; Lobanov et al., 2014; Popov and Sobolev, 2008; Tackley and King, 2003; Torrance and Turcotte, 1971; Zhong and Gurnis, 1994). Available analytical and numerical solutions are mostly two-dimensional and include

- 2-D mantle convection with constant and variable viscosity (Hager and O'Connell, 1981; Revenaugh and Parsons, 1987; Blankenbach et al., 1989);

- 2-D thermochemical convection (van Keken et al., 1997);

- 2-D buoyancy-driven flows for strongly varying viscosity (Zhong, 1996; Moresi et al., 1996; Gerya and Yuen, 2003);

- 2-D mechanical and thermomechanical channel and Couette flows for constant and variable viscosity (Turcotte and Schubert, 2002; Gerya and Yuen, 2003; Gerya, 2010);

- 2-D flow around deformable elliptic inclusions (Schmid and Podladchikov, 2003);

- 2-D Rayleigh-Taylor instability (Ramberg, 1968; Kaus and Becker, 2007); 
- 2-D thermomechanical corner flows in subduction zones (van Keken et al., 2008);

- 2-D spontaneous subduction with a free surface (Schmeling et al., 2008);

- 2-D buoyancy-driven flows with a free surface (Crameri et al., 2012);

- 2-D numerical sandbox experiments (Buiter et al., 2006);

- 3-D mantle convection in Cartesian geometry (Busse et al., 1993; Albers, 2000);

- 3-D mantle convection in spherical geometry (Zhong et al., 2008);

- 3-D infinitesimal and finite amplitude folding instability (Kaus and Schmalholz, 2006);

- 2-D and 3-D shear band formation and plasticity implementation (Lemiale et al., 2008; Kaus, 2010; Thieulot et al., 2008);

- numerical sandbox experiments (Buiter et al., 2006), (Gerya and Yuen, 2007), (Thieulot, 2011) and (Gerya, 2010);

- 3-D convection at infinite Prandtl numbers in Cartesian geometry (Busse et al., 1993);

- falling block (Gerya and Yuen, 2003; Gerya, 2010; Thieulot, 2011).

These solutions are constrained for a number of well defined model setups, which are of potential significance for various situations which numerical codes may face during real geodynamic simulations. Availability and broad range of 2$\mathrm{D}$ and 3-D benchmark solutions are, therefore, critical for the development and testing of the next generation of numerical geodynamic modeling software which aims to combine rheological complexity of constitutive laws with adaptive grid resolution to on both global and regional scales (e.g., Moresi et al., 2003; Dabrowski et al., 2008; Tackley, 2008; Stadler et al., 2010; Gerya et al., 2013).

In the present paper we aim to significantly expand availability of benchmark solutions for both 2-D and 3-D variableviscosity Stokes flows. In contrast with previous studies, we prefer not to start from any prescribed model setups but rather derive general analytical solutions, which are potentially suitable for generating a broad range of test problems. We derive generalized solutions for incompressible Stokes problems with linearly and exponentially variable viscosity. In the following we demonstrate how these generalized solutions can be converted into 2-D and 3-D test problems suitable for benchmarking numerical codes. Finally, based on the obtained benchmark problems, we show examples of numerical convergence tests for staggered-grid discretizations schemes (e.g., Gerya and Yuen, 2003, 2007).

\section{Two-dimensional solution}

\subsection{Formulation of 2-D equations with variable viscosity}

Consider the plane flow, 2-D Stokes equations for the case of varying viscosity have the form

$$
\begin{array}{r}
2 \eta \frac{\partial^{2} v_{x}}{\partial x^{2}}+2 \frac{\partial \eta}{\partial x} \frac{\partial v_{x}}{\partial x}+\eta \frac{\partial^{2} v_{x}}{\partial y^{2}}+\eta \frac{\partial^{2} v_{y}}{\partial y \partial x}+\frac{\partial \eta}{\partial y} \frac{\partial v_{x}}{\partial y} \\
+\frac{\partial \eta}{\partial y} \frac{\partial v_{y}}{\partial x}-\frac{\partial P}{\partial x}=-\rho G_{x}, \\
\eta \frac{\partial^{2} v_{y}}{\partial x^{2}}+\eta \frac{\partial^{2} v_{x}}{\partial y \partial x}+\frac{\partial \eta}{\partial x} \frac{\partial v_{x}}{\partial y}+\frac{\partial \eta}{\partial x} \frac{\partial v_{y}}{\partial x}+2 \frac{\partial \eta}{\partial y} \frac{\partial v_{y}}{\partial y} \\
+2 \eta \frac{\partial^{2} v_{y}}{\partial y^{2}}-\frac{\partial P}{\partial y}=-\rho G_{y}, \\
\frac{\partial v_{x}}{\partial x}+\frac{\partial v_{y}}{\partial y}=0 .
\end{array}
$$

Here $\left(v_{x}, v_{y}\right)$ is the flow velocity, $\eta=\eta(x, y)$ is the viscosity, $P$ is the pressure, $\rho$ is the density, $\left(G_{x}, G_{y}\right)$ is the gravitational force. Note that Eq. (3) is the continuity equation.

Let us change the variables $v_{x}, v_{y}, P$ in such a way that

$$
\begin{array}{ll}
\frac{\partial v_{x}}{\partial x}=\frac{1}{\eta} \frac{\partial u_{x}}{\partial x}, & \frac{\partial v_{x}}{\partial y}=\frac{1}{\eta} \frac{\partial u_{x}}{\partial y}, \\
\frac{\partial v_{y}}{\partial x}=\frac{1}{\eta} \frac{\partial u_{y}}{\partial x}, & \frac{\partial v_{y}}{\partial y}=\frac{1}{\eta} \frac{\partial u_{y}}{\partial y}, \\
\frac{1}{\eta} \frac{\partial P}{\partial x}=\frac{\partial P}{\partial x}, & \frac{1}{\eta} \frac{\partial P}{\partial y}=\frac{\partial \tilde{P}}{\partial y} .
\end{array}
$$

The correctness conditions for such replacement are as follows:

$$
\begin{aligned}
& \frac{\partial}{\partial y}\left(\frac{1}{\eta} \frac{\partial u_{x}}{\partial x}\right)=\frac{\partial}{\partial x}\left(\frac{1}{\eta} \frac{\partial u_{x}}{\partial y}\right), \\
& \frac{\partial}{\partial y}\left(\frac{1}{\eta} \frac{\partial u_{y}}{\partial x}\right)=\frac{\partial}{\partial x}\left(\frac{1}{\eta} \frac{\partial u_{y}}{\partial y}\right), \\
& \frac{\partial}{\partial y}\left(\eta \frac{\partial \tilde{P}}{\partial x}\right)=\frac{\partial}{\partial x}\left(\eta \frac{\partial \tilde{P}}{\partial y}\right) .
\end{aligned}
$$

These conditions lead to the following correlations:

$$
\begin{aligned}
& \frac{\partial \eta}{\partial y} \frac{\partial u_{x}}{\partial x}=\frac{\partial \eta}{\partial x} \frac{\partial u_{x}}{\partial y}, \quad \frac{\partial \eta}{\partial y} \frac{\partial u_{y}}{\partial x}=\frac{\partial \eta}{\partial x} \frac{\partial u_{y}}{\partial y}, \\
& \frac{\partial \eta}{\partial y} \frac{\partial \tilde{P}}{\partial x}=\frac{\partial \eta}{\partial x} \frac{\partial \tilde{P}}{\partial y} .
\end{aligned}
$$

If we consider these conditions as a partial differential equations then we obtain the same characteristic equation for all these conditions:

$$
\frac{\partial \eta}{\partial x} \mathrm{~d} x+\frac{\partial \eta}{\partial y} \mathrm{~d} y=0 .
$$


Evidently, $\eta(x, y)=C$ is an integral of the equation. It is well known that an integral of the characteristic equation gives one good new variable. Namely, $\eta=\eta(x, y)$ is a good new variable (the second coordinate should be orthogonal to the first one). Note that the assumption that the viscosity varies is now crucial because we use the viscosity as a new coordinate (instead of the standard Cartesian spatial coordinate). Hence, the solutions of our equations, which predetermine the correctness of the replacement suggested above, are

$u_{x}=\Phi(\eta), \quad u_{y}=\Psi(\eta), \quad \tilde{P}=\tilde{P}(\eta)$.

After the replacement, the Stokes Eqs. $(1,2)$ and the continuity condition (3) transform to the following form:

$2 \frac{\partial^{2} u_{x}}{\partial x^{2}}+\frac{\partial^{2} u_{x}}{\partial y^{2}}+\frac{\partial^{2} u_{y}}{\partial y \partial x}-\eta \frac{\partial \tilde{P}}{\partial x}=-\rho G_{x}$,

$\frac{\partial^{2} u_{y}}{\partial x^{2}}+\frac{\partial^{2} u_{x}}{\partial y \partial x}+2 \frac{\partial^{2} u_{y}}{\partial y^{2}}-\eta \frac{\partial \tilde{P}}{\partial y}=-\rho G_{y}$,

$\frac{\partial u_{x}}{\partial x}+\frac{\partial u_{y}}{\partial y}=0$.

Inserting the expressions for $u_{x}, u_{y}$ into Eqs. (7-9), one obtains the following equations:

$2 \Phi^{\prime} \frac{\partial^{2} \eta}{\partial^{2} x}+2 \Phi^{\prime \prime}\left(\frac{\partial \eta}{\partial x}\right)^{2}+\Phi^{\prime} \frac{\partial^{2} \eta}{\partial^{2} y}+\Phi^{\prime \prime}\left(\frac{\partial \eta}{\partial y}\right)^{2}+$

$\Psi^{\prime} \frac{\partial^{2} \eta}{\partial y \partial x}+\Psi^{\prime \prime} \frac{\partial \eta}{\partial y} \frac{\partial \eta}{\partial x}-\eta \tilde{P}^{\prime} \frac{\partial \eta}{\partial x}=-\rho G_{x}$,

$2 \Psi^{\prime} \frac{\partial^{2} \eta}{\partial^{2} y}+2 \Psi^{\prime \prime}\left(\frac{\partial \eta}{\partial y}\right)^{2}+\Psi^{\prime} \frac{\partial^{2} \eta}{\partial^{2} x}+\Psi^{\prime \prime}\left(\frac{\partial \eta}{\partial x}\right)^{2}+$

$\Phi^{\prime} \frac{\partial^{2} \eta}{\partial y \partial x}+\Phi^{\prime \prime} \frac{\partial \eta}{\partial y} \frac{\partial \eta}{\partial x}-\eta \tilde{P}^{\prime} \frac{\partial \eta}{\partial y}=-\rho G_{y}$,

$\Phi^{\prime} \frac{\partial \eta}{\partial x}+\Psi^{\prime} \frac{\partial \eta}{\partial y}=0$

\subsection{Linearly varying viscosity}

To obtain the solutions of Eqs. (10-12) we make some assumptions. In particular, in this section we assume, first, that the viscosity $\eta$ is a linear function of the Cartesian coordinates,

$\eta=a x+b y+c$,

where $a, b, c$ are non-zero constants. Second, the restriction concerning the gravitational terms takes place: $\rho\left(a G_{y}-\right.$ $\left.b G_{x}\right)$ and $\rho\left(b G_{y}+a G_{x}\right)$ are assumed to be functions of one variable - viscosity. It means that $\rho G_{y}$ and $\rho G_{x}$ are functions of $\eta$ only. Introduce functions $f, f_{1}$ :

$f(\eta)=\frac{\rho\left(a G_{y}-b G_{x}\right)}{\left(a^{2}+b^{2}\right)^{2}}, \quad f_{1}(\eta)=\frac{\rho\left(b G_{y}+a G_{x}\right)}{a^{2}+b^{2}}$.
Under these assumptions one gets the following system of equations:

$\Phi^{\prime \prime}\left(2 a^{2}+b^{2}\right)+\Psi^{\prime \prime} a b-a \eta \tilde{P}^{\prime}=-\rho G_{x}$,

$\Psi^{\prime \prime}\left(a^{2}+2 b^{2}\right)+\Phi^{\prime \prime} a b-a \eta \tilde{P}=-\rho G_{y}$,

$\Phi^{\prime} a+\Psi^{\prime} b=0$.

One can simply solve this linear algebraic system with respect to $\Phi^{\prime \prime}, \Psi^{\prime \prime}, \tilde{P}^{\prime}$ :

$\Phi^{\prime \prime}=b f(\eta), \quad \Psi^{\prime \prime}=-a f(\eta), \quad \tilde{P}^{\prime}=\frac{f_{1}(\eta)}{\eta}$.

That gives us

$u_{x}=\Phi=b \int_{1}^{\eta} \mathrm{d} \eta_{1} \int_{1}^{\eta_{1}} \mathrm{~d} \eta_{2} f\left(\eta_{2}\right)+b c_{1} \eta+c_{2}$,

$u_{y}=\Psi=-a \int_{1}^{\eta} \mathrm{d} \eta_{1} \int_{1}^{\eta_{1}} \mathrm{~d} \eta_{2} f\left(\eta_{2}\right)-a c_{1} \eta+c_{3}$,

$\tilde{P}=\int_{1}^{\eta} \mathrm{d} \eta_{1} \frac{f_{1}\left(\eta_{1}\right)}{\eta_{1}}+c_{4}$.

Correspondingly, one obtains $v_{x}, v_{y}, P$ :

$$
\begin{aligned}
& v_{x}=b \int_{1}^{\eta} \frac{\mathrm{d} \eta_{1}}{\eta_{1}} \int_{1}^{\eta_{1}} \mathrm{~d} \eta_{2} f\left(\eta_{2}\right)+b c_{1} \log \eta+c_{2} \\
& =b \int_{1}^{\eta} \mathrm{d} \eta_{2} f\left(\eta_{2}\right) \int_{\eta_{2}}^{\eta} \frac{\mathrm{d} \eta_{1}}{\eta_{1}}+b c_{1} \log \eta+c_{2} .
\end{aligned}
$$

Finally,

$v_{x}=b \int_{1}^{\eta} \mathrm{d} \eta_{2} f\left(\eta_{2}\right) \log \left(\frac{\eta}{\eta_{2}}\right)+b c_{1} \log \eta+c_{2}$.

Analogous transformation takes place for $v_{y}$ :

$v_{y}=-a \int_{1}^{\eta} \frac{\mathrm{d} \eta_{1}}{\eta_{1}} \int_{1}^{\eta_{1}} \mathrm{~d} \eta_{2} f\left(\eta_{2}\right)-a c_{1} \log \eta+c_{3}$,

Namely,

$v_{y}=-a \int_{1}^{\eta} \mathrm{d} \eta_{2} f\left(\eta_{2}\right) \log \left(\frac{\eta}{\eta_{2}}\right)-a c_{1} \log \eta+c_{3}$.

The expression for the pressure is as follows:

$P=\int_{1}^{\eta} \mathrm{d} \eta_{1} f_{1}\left(\eta_{1}\right)+c_{4}$ 
In particular, in the case of constant gravitational terms, i.e., for $f(\eta)=A=$ const, $f_{1}(\eta)=A_{1}=$ const one has

$v_{x}=b A \eta+b \tilde{c}_{1} \log \eta+\tilde{c}_{2}$,

$v_{y}=-a A \eta-a \tilde{c}_{1} \log \eta+\tilde{c}_{3}$,

$P=A_{1} \eta+\tilde{c}_{4}$.

Consider a more complicated case when the density is a linear function of the viscosity: $\rho=\beta_{1} \eta+\beta_{2}$. Then,

$f(\eta)=a_{1} \eta+a_{2}, \quad f_{1}(\eta)=b_{1} \eta+b_{2}$,

where constants $a_{1}, a_{2}, b_{1}, b_{2}$ are as follows:

$a_{1}=\beta_{1} \frac{\left(a G_{y}-b G_{x}\right)}{\left(a^{2}+b^{2}\right)^{2}}, \quad a_{2}=\beta_{2} \frac{\left(a G_{y}-b G_{x}\right)}{\left(a^{2}+b^{2}\right)^{2}}$,

$b_{1}=\beta_{1} \frac{\left(b G_{y}+a G_{x}\right)}{a^{2}+b^{2}}, \quad b_{2}=\beta_{2} \frac{\left(b G_{y}+a G_{x}\right)}{a^{2}+b^{2}}$.

Note that in this case the continuity Eq. (3) should be rewritten in a more general form:

$\frac{\partial\left(\rho v_{x}\right)}{\partial x}+\frac{\partial\left(\rho v_{y}\right)}{\partial y}=0$.

Equations (16)-(18) gives us

$$
\begin{array}{r}
v_{x}=-b\left(a_{1} / 2+a_{2}-c_{1}\right) \log \eta+\frac{1}{4} b a_{1} \eta^{2}+b a_{2} \eta \\
-\frac{1}{4} a_{1} b-a_{2} b+c_{2}, \\
v_{y}=a\left(a_{1} / 2+a_{2}-c_{1}\right) \log \eta-\frac{1}{4} a a_{1} \eta^{2}-a a_{2} \eta \\
+\frac{1}{4} a_{1} a+a_{2} a+c_{3}, \\
P=\frac{1}{2} b_{1} \eta^{2}+b_{2} \eta-\frac{1}{2} b_{1}-b_{2}+c_{4} .
\end{array}
$$

The continuity Eq. (19) gives us the relation between $c_{2}, c_{3}$ : $a c_{2}+b c_{3}=0$.

\subsection{Exponentially varying viscosity}

Let us construct the second benchmark solution. Now we assume that the viscosity is the exponential function of the Cartesian coordinates:

$\eta=c \exp (a x+b y)$

General consideration up to Eqs. (10-12) is the same as earlier. By inserting Eq. (20) into Eqs. (10-12) and taking into account that

$$
\frac{\partial \eta}{\partial x}=a \eta, \quad \frac{\partial \eta}{\partial y}=b \eta,
$$

one obtains the following system of equations:

$$
\begin{aligned}
& \left(2 a^{2}+b^{2}\right)\left(\Phi^{\prime \prime} \eta^{2}+\Phi^{\prime} \eta\right)+a b\left(\Psi^{\prime \prime} \eta^{2}+\Psi^{\prime} \eta\right) \\
& -a \tilde{P}^{\prime} \eta^{2}=-\rho G_{x}, \\
& a b\left(\Phi^{\prime \prime} \eta^{2}+\Phi^{\prime} \eta\right)+\left(a^{2}+2 b^{2}\right)\left(\Psi^{\prime \prime} \eta^{2}+\Psi^{\prime} \eta\right) \\
& -b \tilde{P}^{\prime} \eta^{2}=-\rho G_{y}, \\
& a \Phi^{\prime}+b \Psi^{\prime}=0 .
\end{aligned}
$$

Using the last relation, we exclude $\Psi$ from the first two equations:

$$
\begin{aligned}
& \left(a^{2}+b^{2}\right)\left(\Phi^{\prime \prime} \eta^{2}+\Phi^{\prime} \eta\right)-a \tilde{P}^{\prime} \eta^{2}=-\rho G_{x}, \\
& -\frac{a^{3}+a b^{2}}{b}\left(\Phi^{\prime \prime} \eta^{2}+\Phi^{\prime} \eta\right)-b \tilde{P}^{\prime} \eta^{2}=-\rho G_{y}, \\
& \Psi^{\prime}=-\frac{a}{b} \Phi^{\prime} .
\end{aligned}
$$

One can see that we obtain a linear algebraic system with respect to $\left(\Phi^{\prime \prime} \eta^{2}+\Phi^{\prime} \eta\right)$ and $\tilde{P}^{\prime}$. The solution is as follows:

$$
\begin{aligned}
& \tilde{P}^{\prime}=\frac{f_{1}(\eta)}{\eta^{2}}, \\
& \Phi^{\prime \prime} \eta^{2}+\Phi^{\prime} \eta=b f(\eta) .
\end{aligned}
$$

Remark. It is interesting that these formulas contain the same functions $f(\eta), f_{1}(\eta)$ as in the previous section.

Equation (23) is a well-known Euler ordinary differential equation. One can get its solution for arbitrary function $f$ :

$u_{x}=\Phi(\eta)=b \int_{1}^{\eta} \log \left(\frac{\eta}{\eta_{1}}\right) \frac{f\left(\eta_{1}\right)}{\eta_{1}} d \eta_{1}+b c_{1} \log \eta+c_{2}$.

Taking into account the relation in Eq. (21), one obtains $u_{y}$ :

$\left.u_{y}=\Psi(\eta)=-a \int_{1}^{\eta} \log \left(\frac{\eta}{\eta_{1}}\right) \frac{f\left(\eta_{1}\right)}{\eta_{1}}\right) d \eta_{1}-a c_{1} \log \eta+c_{3}$.

Taking into account Eqs. $(4,5)$ one obtains $v_{x}, v_{y}$ :

$$
\begin{aligned}
v_{x} & =b \int_{1}^{\eta} \frac{d \eta_{1}}{\eta_{1}^{2}} \int_{1}^{\eta_{1}} d \eta_{2} \frac{f\left(\eta_{2}\right)}{\eta_{2}}-b c_{1} \frac{1}{\eta}+b c_{1}+c_{2} \\
& =b \int_{1}^{\eta} d \eta_{2} \frac{f\left(\eta_{2}\right)}{\eta_{2}} \int_{\eta_{2}}^{\eta} \frac{d \eta_{1}}{\eta_{1}^{2}}-b c_{1} \frac{1}{\eta}+b c_{1}+c_{2} .
\end{aligned}
$$

Hence, we get the expression for $v_{x}$ and analogously, for $v_{y}$ :

$$
\begin{aligned}
& v_{x}=b \int_{1}^{\eta} d \eta_{2} \frac{f\left(\eta_{2}\right)}{\eta_{2}} \frac{\eta-\eta_{2}}{\eta \eta_{2}}-b c_{1} \frac{1}{\eta}+b c_{1}+c_{2}, \\
& v_{y}=-a \int_{1}^{\eta} d \eta_{2} \frac{f\left(\eta_{2}\right)}{\eta_{2}} \frac{\eta-\eta_{2}}{\eta \eta_{2}}+a c_{1} \frac{1}{\eta}-a c_{1}+c_{3} .
\end{aligned}
$$


As for the pressure, we obtain it from Eq. (22) by taking into account Eq. (6):

$\tilde{P}=\int_{1}^{\eta} d \eta_{1} \frac{f_{1}\left(\eta_{1}\right)}{\eta_{1}^{2}}+c_{4}$.

Hence,

$P=\int_{1}^{\eta} d \eta_{1} \frac{f_{1}\left(\eta_{1}\right)}{\eta_{1}}+c_{4}$.

One can compare Eqs. (26-28) with Eqs. (16-18).

For a simple particular case (constant gravitational term) when $f(\eta)=A$ = const, $f_{1}(\eta)=A_{1}=$ const one has

$v_{x}=-\frac{b\left(A+c_{1}\right)}{\eta}-\frac{b A \log \eta}{\eta}+\tilde{c}_{2}$,

$v_{y}=\frac{a\left(A+c_{1}\right)}{\eta}+\frac{a A \log \eta}{\eta}+\tilde{c}_{3}$,

$P=A_{1} \log \eta+c_{4}-b_{1}$,

where $\tilde{c}_{2}=b c_{1}+c_{2}, \tilde{c}_{3}=-a c_{1}+c_{3}$.

For more a complicated case when the density is a linear function of the viscosity $\rho=\beta_{1} \eta+\beta_{2}$, i.e.,

$f(\eta)=a_{1} \eta+a_{2}, \quad f_{1}(\eta)=b_{1} \eta+b_{2}$,

where constants $a_{1}, a_{2}, b_{1}, b_{2}$ are the same as in the previous section. The continuity equation should be written in a more general form Eq. (19). It is simple to evaluate integrals in Eqs. (26-28). In such a way one obtains

$v_{x}=b a_{1} \log \eta+\frac{b\left(a_{1}-a_{2}-c_{1}\right)}{\eta}-b a_{2} \frac{\log \eta}{\eta}+\tilde{c}_{2}$,

$v_{y}=-a a_{1} \log \eta-\frac{a\left(a_{1}-a_{2}-c_{1}\right)}{\eta}+a a_{2} \frac{\log \eta}{\eta}+\tilde{c}_{3}$,

$P=b_{1} \eta+b_{2} \log \eta+\tilde{c}_{4}$,

where $\quad \tilde{c}_{2}=c_{2}+b c_{1}+b a_{2}-b a_{1}, \tilde{c}_{3}=c_{3}+a a_{1}-a a_{2}-$ $a c_{1}, \tilde{c}_{4}=c_{4}-b_{1}$. The continuity Eq. (19) gives us the same relation as earlier:

$a c_{2}+b c_{3}=0$.

\section{Three-dimensional case}

\subsection{Formulation of 3-D equations with variable viscosity}

The situation in the 3-D case is similar to that for 2-D. In particular, we can realize the same procedure as in the 2-D case with some additional restrictions. The initial system of equations is as follows:

$2 \eta \frac{\partial^{2} v_{x}}{\partial x^{2}}+2 \frac{\partial \eta}{\partial x} \frac{\partial v_{x}}{\partial x}+\eta \frac{\partial^{2} v_{x}}{\partial y^{2}}+\eta \frac{\partial^{2} v_{y}}{\partial y \partial x}+\frac{\partial \eta}{\partial y} \frac{\partial v_{x}}{\partial y}+\frac{\partial \eta}{\partial y} \frac{\partial v_{y}}{\partial x}$
$+\eta \frac{\partial^{2} v_{x}}{\partial z^{2}}+\eta \frac{\partial^{2} v_{z}}{\partial z \partial x}+\frac{\partial \eta}{\partial z} \frac{\partial v_{x}}{\partial z}+\frac{\partial \eta}{\partial z} \frac{\partial v_{z}}{\partial x}-\frac{\partial P}{\partial x}=-\rho G_{x}$,
$\eta \frac{\partial^{2} v_{y}}{\partial x^{2}}+\eta \frac{\partial^{2} v_{x}}{\partial y \partial x}+\frac{\partial \eta}{\partial x} \frac{\partial v_{x}}{\partial y}+\frac{\partial \eta}{\partial x} \frac{\partial v_{y}}{\partial x}+2 \frac{\partial \eta}{\partial y} \frac{\partial v_{y}}{\partial y}+2 \eta \frac{\partial^{2} v_{y}}{\partial y^{2}}$
$+\eta \frac{\partial^{2} v_{y}}{\partial z^{2}}+\eta \frac{\partial^{2} v_{z}}{\partial z \partial y}+\frac{\partial \eta}{\partial z} \frac{\partial v_{y}}{\partial z}+\frac{\partial \eta}{\partial z} \frac{\partial v_{z}}{\partial y}-\frac{\partial P}{\partial y}=-\rho G_{y}$,
$\eta \frac{\partial^{2} v_{z}}{\partial x^{2}}+\eta \frac{\partial^{2} v_{x}}{\partial z \partial x}+\frac{\partial \eta}{\partial x} \frac{\partial v_{z}}{\partial x}+\frac{\partial \eta}{\partial x} \frac{\partial v_{x}}{\partial z}+\eta \frac{\partial^{2} v_{y}}{\partial z \partial y}+\frac{\partial \eta}{\partial y} \frac{\partial v_{z}}{\partial y}$
$+\frac{\partial \eta}{\partial y} \frac{\partial v_{y}}{\partial z}+\eta \frac{\partial^{2} v_{z}}{\partial y^{2}}+2 \eta \frac{\partial^{2} v_{z}}{\partial z^{2}}+2 \frac{\partial \eta}{\partial z} \frac{\partial v_{z}}{\partial z}-\frac{\partial P}{\partial z}=-\rho G_{z}$,
$\frac{\partial v_{x}}{\partial x}+\frac{\partial v_{y}}{\partial y}+\frac{\partial v_{z}}{\partial z}=0$.

The replacement of the variables $v_{x}, v_{y}, v_{z}, P$ by $u_{x}, u_{y}, u_{z}, \tilde{P}$ is analogous to that in the two-dimensional case:

$\frac{\partial v_{x}}{\partial x}=\frac{1}{\eta} \frac{\partial u_{x}}{\partial x}, \quad \frac{\partial v_{x}}{\partial y}=\frac{1}{\eta} \frac{\partial u_{x}}{\partial y}$,

$\frac{\partial v_{x}}{\partial z}=\frac{1}{\eta} \frac{\partial u_{x}}{\partial z}, \quad \frac{\partial v_{y}}{\partial x}=\frac{1}{\eta} \frac{\partial u_{y}}{\partial x}$,

$\frac{\partial v_{y}}{\partial y}=\frac{1}{\eta} \frac{\partial u_{y}}{\partial y}, \quad \frac{\partial v_{y}}{\partial z}=\frac{1}{\eta} \frac{\partial u_{y}}{\partial z}$,

$\frac{\partial v_{z}}{\partial x}=\frac{1}{\eta} \frac{\partial u_{z}}{\partial x}, \quad \frac{\partial v_{z}}{\partial y}=\frac{1}{\eta} \frac{\partial u_{z}}{\partial y}$,

$\frac{\partial v_{z}}{\partial z}=\frac{1}{\eta} \frac{\partial u_{z}}{\partial z}, \quad \frac{1}{\eta} \frac{\partial P}{\partial x}=\frac{\partial \tilde{P}}{\partial x}$,

$\frac{1}{\eta} \frac{\partial P}{\partial y}=\frac{\partial \tilde{P}}{\partial y}, \quad \frac{1}{\eta} \frac{\partial P}{\partial z}=\frac{\partial \tilde{P}}{\partial z}$.

The correctness conditions for such replacement are as follows:

$$
\begin{array}{ll}
\frac{\partial \eta}{\partial y} \frac{\partial u_{x}}{\partial x}=\frac{\partial \eta}{\partial x} \frac{\partial u_{x}}{\partial y}, & \frac{\partial \eta}{\partial z} \frac{\partial u_{x}}{\partial x}=\frac{\partial \eta}{\partial x} \frac{\partial u_{x}}{\partial z}, \\
\frac{\partial \eta}{\partial z} \frac{\partial u_{x}}{\partial y}=\frac{\partial \eta}{\partial y} \frac{\partial u_{x}}{\partial z}, & \frac{\partial \eta}{\partial y} \frac{\partial u_{y}}{\partial x}=\frac{\partial \eta}{\partial x} \frac{\partial u_{y}}{\partial y}, \\
\frac{\partial \eta}{\partial z} \frac{\partial u_{y}}{\partial x}=\frac{\partial \eta}{\partial x} \frac{\partial u_{y}}{\partial z}, & \frac{\partial \eta}{\partial z} \frac{\partial u_{y}}{\partial y}=\frac{\partial \eta}{\partial y} \frac{\partial u_{y}}{\partial z}, \\
\frac{\partial \eta}{\partial y} \frac{\partial u_{z}}{\partial x}=\frac{\partial \eta}{\partial x} \frac{\partial u_{z}}{\partial y}, & \frac{\partial \eta}{\partial z} \frac{\partial u_{z}}{\partial x}=\frac{\partial \eta}{\partial x} \frac{\partial u_{z}}{\partial z}, \\
\frac{\partial \eta}{\partial z} \frac{\partial u_{z}}{\partial y}=\frac{\partial \eta}{\partial y} \frac{\partial u_{z}}{\partial z}, & \frac{\partial \eta}{\partial y} \frac{\partial \tilde{P}}{\partial x}=\frac{\partial \eta}{\partial x} \frac{\partial \tilde{P}}{\partial y}, \\
\frac{\partial \eta}{\partial y} \frac{\partial \tilde{P}}{\partial x}=\frac{\partial \eta}{\partial x} \frac{\partial \tilde{P}}{\partial y}, & \frac{\partial \eta}{\partial z} \frac{\partial \tilde{P}}{\partial x}=\frac{\partial \eta}{\partial x} \frac{\partial \tilde{P}}{\partial z} .
\end{array}
$$


The characteristic equations for each triplet of equations, i.e., for $u_{x}, u_{y}, u_{z}, \tilde{P}$ are the same (analogously to 2-D case). The solutions for these conditional equations are

$u_{x}=\Phi(\eta), \quad u_{y}=\Psi(\eta), \quad u_{z}=\Gamma(\eta), \quad \tilde{P}=\tilde{P}(\eta)$

These relations give us correctness of the above introduced replacement for arbitrarily varying viscosity. However, it allows one to obtain the solution of the Stokes continuity equations only under some assumptions concerning the viscosity.

\subsection{Linearly varying viscosity - 3-D case}

Below we will assume (analogously to 2-D case, Eq. 13) that

$\eta=a x+b y+c z+e$,

where $a, b, c, e$ are non-zero constants. Moreover, in the 3-D case we need the following restriction for the gravitational terms (compare with Eq. 14): $\rho G_{x}, \rho G_{y}, \rho G_{z}$ are functions of one variable - viscosity (for example, these terms may be constant).

Making the substitution (Eq. 31) in the system (Eq. 29), one transforms it to the form,

$$
\begin{aligned}
2 \frac{\partial^{2} u_{x}}{\partial x^{2}} & +\frac{\partial^{2} u_{x}}{\partial y^{2}}+\frac{\partial^{2} u_{y}}{\partial y \partial x}+\frac{\partial^{2} u_{x}}{\partial z^{2}} \\
& +\frac{\partial^{2} u_{z}}{\partial z \partial x}-\eta \frac{\partial \tilde{P}}{\partial x}=-\rho G_{x} \\
\frac{\partial^{2} u_{y}}{\partial x^{2}}+ & \frac{\partial^{2} u_{x}}{\partial y \partial x}+2 \frac{\partial^{2} u_{y}}{\partial y^{2}}+\frac{\partial^{2} u_{y}}{\partial z^{2}} \\
& +\frac{\partial^{2} u_{z}}{\partial z \partial y}-\eta \frac{\partial \tilde{P}}{\partial y}=-\rho G_{y} \\
\frac{\partial^{2} u_{z}}{\partial x^{2}} & +\frac{\partial^{2} u_{x}}{\partial z \partial x}+\frac{\partial^{2} u_{y}}{\partial z \partial y}+\frac{\partial^{2} u_{z}}{\partial y^{2}} \\
+ & 2 \frac{\partial^{2} u_{z}}{\partial z^{2}}-\eta \frac{\partial \tilde{P}}{\partial z}=-\rho G_{z} \\
\frac{\partial u_{x}}{\partial x}+ & \frac{\partial u_{y}}{\partial y}+\frac{\partial u_{z}}{\partial z}=0 .
\end{aligned}
$$

Substitution of Eq. (32) into Eq. (??), Eq. (35) leads to the following system for $\Phi, \Psi, \Gamma, \tilde{P}$ :

$$
\begin{aligned}
& \Phi^{\prime \prime}\left(2 a^{2}+b^{2}+c^{2}\right)+\Psi^{\prime \prime} a b+\Gamma^{\prime \prime} a c-\eta a \tilde{P}^{\prime}=-\rho G_{x}, \\
& \Psi^{\prime \prime}\left(a^{2}+2 b^{2}+c^{2}\right)+\Phi^{\prime \prime} a b+\Gamma^{\prime \prime} b c-\eta b \tilde{P}^{\prime}=-\rho G_{y}, \\
& \Gamma^{\prime \prime}\left(a^{2}+b^{2}+2 c^{2}\right)+\Phi^{\prime \prime} a c+\Psi^{\prime \prime} b c-\eta c \tilde{P}^{\prime}=-\rho G_{z}, \\
& \Phi^{\prime} a+\Psi^{\prime} b+\Gamma^{\prime} c=0 .
\end{aligned}
$$

Hence,

$$
\begin{aligned}
& \Phi^{\prime \prime}\left(a^{2}+b^{2}+c^{2}\right)-\eta a \tilde{P}^{\prime}=-\rho G_{x}, \\
& \Psi^{\prime \prime}\left(a^{2}+b^{2}+c^{2}\right)-\eta b \tilde{P}^{\prime}=-\rho G_{y}, \\
& -\frac{1}{c} \Phi^{\prime \prime}\left(a c^{2}+a b^{2}+a^{3}\right)-\frac{1}{c} \Psi^{\prime \prime}\left(b c^{2}+a^{2} b+b^{3}\right) \\
& -\eta c \tilde{P}^{\prime}=-\rho G_{z}, \Gamma^{\prime}=-\frac{a}{c} \Phi^{\prime}-\frac{b}{c} \Psi^{\prime} .
\end{aligned}
$$

The first three equations gives one a linear algebraic system with respect to $\Phi^{\prime \prime}, \Psi^{\prime \prime}, \tilde{P}^{\prime}$ which can be solved without difficulties. Then, one gets $\Gamma^{\prime \prime}$ from the last equation of the system. To be correct, the obtained expressions should be functions of one variable - viscosity. It is ensured by our assumption concerning the gravitational terms. The result is as follows:

$$
\begin{aligned}
& \Phi^{\prime \prime}=\frac{\rho\left(G_{y} a b+G_{z} a c-G_{x}\left(b^{2}+c^{2}\right)\right)}{\left(a^{2}+b^{2}+c^{2}\right)^{2}}=f_{x}(\eta), \\
& \Psi^{\prime \prime}=\frac{\rho\left(G_{x} a b+G_{z} b c-G_{y}\left(a^{2}+c^{2}\right)\right)}{\left(a^{2}+b^{2}+c^{2}\right)^{2}}=f_{y}(\eta), \\
& \Gamma^{\prime \prime}=\frac{\rho\left(G_{x} a c+G_{y} b c-G_{z}\left(a^{2}+b^{2}\right)\right)}{\left(a^{2}+b^{2}+c^{2}\right)^{2}}=f_{z}(\eta), \\
& \tilde{P}^{\prime}=\frac{\rho\left(G_{x} a+G_{y} b+G_{x} c\right)}{\eta\left(a^{2}+b^{2}+c^{2}\right)}=\frac{f_{P}(\eta)}{\eta} .
\end{aligned}
$$

Here we defined four functions: $f_{x}(\eta), f_{y}(\eta), f_{z}(\eta), f_{P}(\eta)$. The obtained expressions are analogous to that in Eq. (15). Correspondingly, the integration is analogous, and one obtains

$$
\begin{aligned}
& v_{x}=\int_{1}^{\eta} d \eta_{2} f_{x}\left(\eta_{2}\right) \log \left(\frac{\eta}{\eta_{2}}\right)+c_{1 x} \log \eta+c_{2 x}, \\
& v_{y}=\int_{1}^{\eta} d \eta_{2} f_{y}\left(\eta_{2}\right) \log \left(\frac{\eta}{\eta_{2}}\right)+c_{1 y} \log \eta+c_{2 y}, \\
& v_{z}=\int_{1}^{\eta} d \eta_{2} f_{z}\left(\eta_{2}\right) \log \left(\frac{\eta}{\eta_{2}}\right)+c_{1 z} \log \eta+c_{2 z}, \\
& P=\int_{1}^{\eta} d \eta_{1} f_{P}\left(\eta_{1}\right)+c_{p} .
\end{aligned}
$$

In particular, in the case of constant gravitational terms, i.e., for $f_{x}(\eta)=A_{x}=$ const, $f_{y}(\eta)=A_{y}=$ const, $f_{z}(\eta)=$ $A_{z}=$ const, $f_{P}(\eta)=A_{P}=$ const one obtains a result analogous to the 2-D case:

$$
\begin{aligned}
& v_{x}=A_{x} \eta+\tilde{c}_{1 x} \log \eta+\tilde{c}_{2 x}, \\
& v_{y}=A_{y} \eta+\tilde{c}_{1 y} \log \eta+\tilde{c}_{2 y}, \\
& v_{z}=A_{z} \eta+\tilde{c}_{1 z} \log \eta+\tilde{c}_{2 z}, \\
& P=A_{P} \eta+\tilde{c}_{P} .
\end{aligned}
$$


The continuity condition gives one the following correlation between the coefficients:

$a \tilde{c}_{1 x}+b \tilde{c}_{1 y}+c \tilde{c}_{1 z}=0$.

The condition,

$a A_{x}+b A_{y}+c A_{z}=0$,

is identically valid (see the expressions for $f_{x}, f_{y}, f_{z}$ ).

Consider a more complicated case when the density is a linear function of the viscosity: $\rho=\beta_{1} \eta+\beta_{2}$. Then,

$f_{x}(\eta)=a_{1} \eta+a_{2}, \quad f_{y}(\eta)=b_{1} \eta+b_{2}$,

$f_{z}(\eta)=d_{1} \eta+d_{2}, \quad f_{P}(\eta)=p_{1} \eta+p_{2}$,

where constants $a_{1}, a_{2}, b_{1}, b_{2}, d_{1}, d_{2}, p_{1}, p_{2}$ are as follows:

$$
\begin{aligned}
& a_{1}=\beta_{1} \frac{\left(G_{y} a b+G_{z} a c-G_{x}\left(b^{2}+c^{2}\right)\right)}{\left(a^{2}+b^{2}+c^{2}\right)^{2}}, \\
& a_{2}=\beta_{2} \frac{\left(G_{y} a b+G_{z} a c-G_{x}\left(b^{2}+c^{2}\right)\right)}{\left(a^{2}+b^{2}+c^{2}\right)^{2}}, \\
& b_{1}=\beta_{1} \frac{\left(G_{x} a b+G_{z} b c-G_{y}\left(a^{2}+c^{2}\right)\right)}{\left(a^{2}+b^{2}+c^{2}\right)^{2}}, \\
& b_{2}=\beta_{2} \frac{\left(G_{x} a b+G_{z} b c-G_{y}\left(a^{2}+c^{2}\right)\right)}{\left(a^{2}+b^{2}+c^{2}\right)^{2}}, \\
& d_{1}=\beta_{1} \frac{\left(G_{x} a c+G_{y} b c-G_{z}\left(a^{2}+b^{2}\right)\right)}{\left(a^{2}+b^{2}+c^{2}\right)^{2}}, \\
& d_{2}=\beta_{2} \frac{\left(G_{x} a c+G_{y} b c-G_{z}\left(a^{2}+b^{2}\right)\right)}{\left(a^{2}+b^{2}+c^{2}\right)^{2}}, \\
& p_{1}=\beta_{1} \frac{\left(G_{x} a+G_{y} b+G_{x} c\right)}{\left(a^{2}+b^{2}+c^{2}\right)}, \\
& p_{2}=\beta_{2} \frac{\left(G_{x} a+G_{y} b+G_{x} c\right)}{\left(a^{2}+b^{2}+c^{2}\right)} .
\end{aligned}
$$

The continuity Eq. (30) in this situation has more general form:

$$
\frac{\partial\left(\rho v_{x}\right)}{\partial x}+\frac{\partial\left(\rho v_{y}\right)}{\partial y}+\frac{\partial\left(\rho v_{z}\right)}{\partial z}=0 .
$$

In this case the formulas for the velocity and the pressure preserve their form from Eqs. (44-47) and give us

$$
\begin{array}{r}
v_{x}=-\left(a_{1} / 2+a_{2}-c_{1 x}\right) \log \eta+\frac{1}{4} a_{1} \eta^{2}+a_{2} \eta-\frac{1}{4} a_{1} \\
-a_{2}+c_{2 x}, \\
v_{y}=-\left(b_{1} / 2+b_{2}-c_{1 y}\right) \log \eta+\frac{1}{4} b_{1} \eta^{2}+b_{2} \eta-\frac{1}{4} b_{1} \\
-b_{2}+c_{2 y}, \\
v_{z}=-\left(d_{1} / 2+d_{2}-c_{1 z}\right) \log \eta+\frac{1}{4} d_{1} \eta^{2}+d_{2} \eta-\frac{1}{4} d_{1} \\
-d_{2}+c_{2 z}, \\
P=\frac{1}{2} p_{1} \eta^{2}+p_{2} \eta-\frac{1}{2} p_{1}-p_{2}+c_{p} .
\end{array}
$$

The continuity Eq. (49) leads to the relation

$a c_{2 x}+b c_{2 y}+c c_{2 z}=0$.

\subsection{Exponentially varying viscosity - 3-D case}

Consider the second benchmark solution (for exponential dependence of the viscosity on the Cartesian coordinates):

$\eta=C \exp (a x+b y+c z)$.

The assumption concerning the gravitational terms is the same as earlier. General 3-D consideration is the same. By inserting Eq. (32) into the equations for $u_{x}, u_{y}, u_{z}, \tilde{P}$ and taking into account that

$\frac{\partial \eta}{\partial x}=a \eta, \quad \frac{\partial \eta}{\partial y}=b \eta, \quad \frac{\partial \eta}{\partial z}=c \eta$,

one obtains the following system of equations:

$$
\begin{aligned}
& \left(2 a^{2}+b^{2}+c^{2}\right)\left(\Phi^{\prime \prime} \eta^{2}+\Phi^{\prime} \eta\right)+a b\left(\Psi^{\prime \prime} \eta^{2}+\Psi^{\prime} \eta\right) \\
& +a c\left(\Gamma^{\prime \prime} \eta^{2}+\Gamma^{\prime} \eta\right)-a \tilde{P}^{\prime} \eta^{2}=-\rho G_{x}, \\
& a b\left(\Phi^{\prime \prime} \eta^{2}+\Phi^{\prime} \eta\right)+\left(a^{2}+2 b^{2}+c^{2}\right)\left(\Psi^{\prime \prime} \eta^{2}+\Psi^{\prime} \eta\right) \\
& +b c\left(\Gamma^{\prime \prime} \eta^{2}+\Gamma^{\prime} \eta\right)-b \tilde{P}^{\prime} \eta^{2}=-\rho G_{y}, \\
& a c\left(\Phi^{\prime \prime} \eta^{2}+\Phi^{\prime} \eta\right)+b c\left(\Psi^{\prime \prime} \eta^{2}+\Psi^{\prime} \eta\right)+\left(a^{2}+b^{2}+2 c^{2}\right) \\
& \left(\Gamma^{\prime \prime} \eta^{2}+\Gamma^{\prime} \eta\right)-c \tilde{P}^{\prime} \eta^{2}=-\rho G_{z}, \\
& a \Phi^{\prime}+b \Psi^{\prime}+c \Gamma^{\prime}=0 .
\end{aligned}
$$

We can note that the first three equations give us the same linear algebraic system as in the case of linear viscosity (Eqs. 36-38), if one takes $\left(\Phi^{\prime \prime} \eta^{2}+\Phi^{\prime} \eta\right),\left(\Psi^{\prime \prime} \eta^{2}+\Psi^{\prime} \eta\right)$, $\left(\Gamma^{\prime \prime} \eta^{2}+\Gamma^{\prime} \eta\right), \eta \tilde{P}^{\prime}$ as variables instead of $\Phi^{\prime \prime}, \Psi^{\prime \prime}, \Gamma^{\prime \prime}, \tilde{P}$ in the linear viscosity case. Hence, the solution of the system is as follows:

$$
\begin{aligned}
& \Phi^{\prime \prime} \eta^{2}+\Phi^{\prime} \eta=f_{x}(\eta), \\
& \Psi^{\prime \prime} \eta^{2}+\Psi^{\prime} \eta=f_{y}(\eta), \\
& \Gamma^{\prime \prime} \eta^{2}+\Gamma^{\prime} \eta=f_{z}(\eta), \\
& \tilde{P}^{\prime} \eta=\frac{f_{P}(\eta)}{\eta} .
\end{aligned}
$$

The definition of the functions $f_{x}, f_{y}, f_{z}, f_{p}$ was given above, see Eqs. (40-43). One can solve these equations by the procedure used in the corresponding 2-D case (exponential viscosity). By this method, we come to the result

$$
\begin{aligned}
& v_{x}=\int_{1}^{\eta} d \eta_{2} \frac{f_{x}\left(\eta_{2}\right)}{\eta_{2}} \frac{\eta-\eta_{2}}{\eta \eta_{2}}+c_{1 x} \frac{1}{\eta}+c_{2 x}, \\
& v_{y}=\int_{1}^{\eta} d \eta_{2} \frac{f_{y}\left(\eta_{2}\right)}{\eta_{2}} \frac{\eta-\eta_{2}}{\eta \eta_{2}}+c_{1 y} \frac{1}{\eta}+c_{2 y} . \\
& v_{z}=\int_{1}^{\eta} d \eta_{2} \frac{f_{z}\left(\eta_{2}\right)}{\eta_{2}} \frac{\eta-\eta_{2}}{\eta \eta_{2}}+c_{1 z} \frac{1}{\eta}+c_{2 z} . \\
& P=\int_{1}^{\eta} d \eta_{1} \frac{f_{P}\left(\eta_{1}\right)}{\eta_{1}}+c_{p} .
\end{aligned}
$$


Here $c_{1 x}, c_{1 y}, c_{1 z}, c_{2 x}, c_{2 y}, c_{2 z}, c_{p}$ are constants. The continuity equation gives one a relation between the coefficients:

$a c_{1 x}+b c_{1 y}+c c_{1 z}=0$.

One can compare Eqs. (51-54) with the results for the corresponding 2-D case (Eqs. 26-28).

For a particularly simple case (constant gravitational term) when $f_{x}(\eta)=A_{x}=$ const, $f_{y}(\eta)=A_{y}=$ const, $f_{z}(\eta)=$ $A_{z}=$ const, $f_{P}(\eta)=A_{P}=$ const one has:

$v_{x}=-\frac{\left(A_{x}+c_{1 x}\right)}{\eta}-A_{x} \frac{\log \eta}{\eta}+\tilde{c}_{2 x}$,

$v_{y}=-\frac{\left(A_{y}+c_{1 y}\right)}{\eta}-A_{y} \frac{\log \eta}{\eta}+\tilde{c}_{2 y}$,

$v_{z}=-\frac{\left(A_{z}+c_{1 z}\right)}{\eta}-A_{z} \frac{\log \eta}{\eta}+\tilde{c}_{2 z}$,

$P=A_{P} \log \eta+c_{p}$.

Consider a more complicated case when the density is a linear function of the viscosity: $\rho=\beta_{1} \eta+\beta_{2}$. Then,

$$
\begin{array}{ll}
f_{x}(\eta)=a_{1} \eta+a_{2}, & f_{y}(\eta)=b_{1} \eta+b_{2}, \\
f_{z}(\eta)=d_{1} \eta+d_{2}, & f_{P}(\eta)=p_{1} \eta+p_{2},
\end{array}
$$

where constants $a_{1}, a_{2}, b_{1}, b_{2}, d_{1}, d_{2}, p_{1}, p_{2}$ have been determined in the previous section. Here we use the continuity equation in the form of Eq. (49). In this case Eqs. (51-54) give us

$$
\begin{aligned}
& v_{x}=a_{1} \log \eta+\frac{\left(a_{1}-a_{2}-c_{1 x}\right)}{\eta}-a_{2} \frac{\log \eta}{\eta}+\tilde{c}_{2 x}, \\
& v_{y}=b_{1} \log \eta+\frac{\left(b_{1}-b_{2}-c_{1 y}\right)}{\eta}-b_{2} \frac{\log \eta}{\eta}+\tilde{c}_{2 y}, \\
& v_{z}=d_{1} \log \eta+\frac{\left(d_{1}-d_{2}-c_{1 y}\right)}{\eta}-d_{2} \frac{\log \eta}{\eta}+\tilde{c}_{2 y}, \\
& P=p_{1} \eta+p_{2} \log \eta+\tilde{c}_{P}
\end{aligned}
$$

where $\tilde{c}_{2 x}=c_{2 x}+c_{1 x}+a_{2}-a_{1}, \tilde{c}_{2 y}=c_{2 y}+c_{1 y}+b_{2}-b_{1}$, $\tilde{c}_{2 z}=c_{2 z}+c_{1 z}+d_{2}-d_{1}, \quad \tilde{c}_{P}=c_{p}-p_{1}$. The continuity Eq. (49) leads to the relation

$a \tilde{c}_{2 x}+b \tilde{c}_{2 y}+c \tilde{c}_{2 z}=0$.

\section{Example problems and numerical convergence tests}

The scheme of algorithm testing is as follows. Initially, we have obtained particular solutions of the Stokes and continuity equations for two types of viscosity variations. Let us choose a domain, e.g., a rectangle in the 2-D case. We calculate the values for velocity and pressure given by our analytical solution and take these values as the boundary conditions.
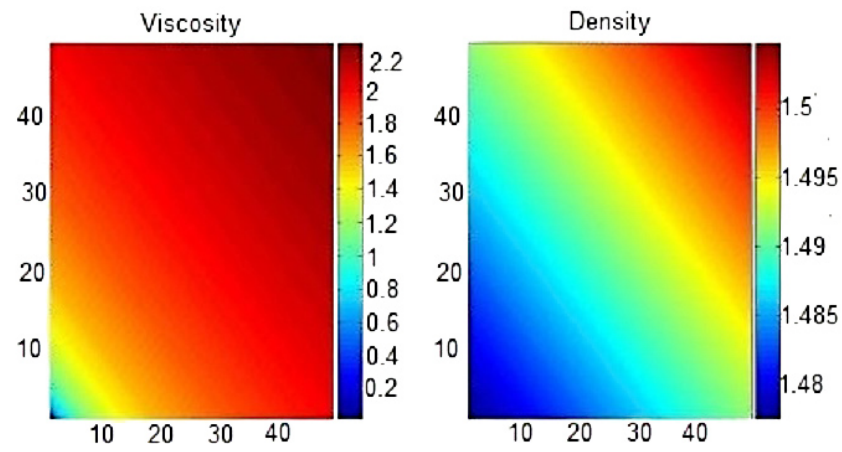

Figure 1. Distribution of viscosity $\eta$ and density $\rho$; 2-D case, linearly varying viscosity, high-viscosity contrast $\left(\eta_{2}=\eta_{3}=100\right)$.
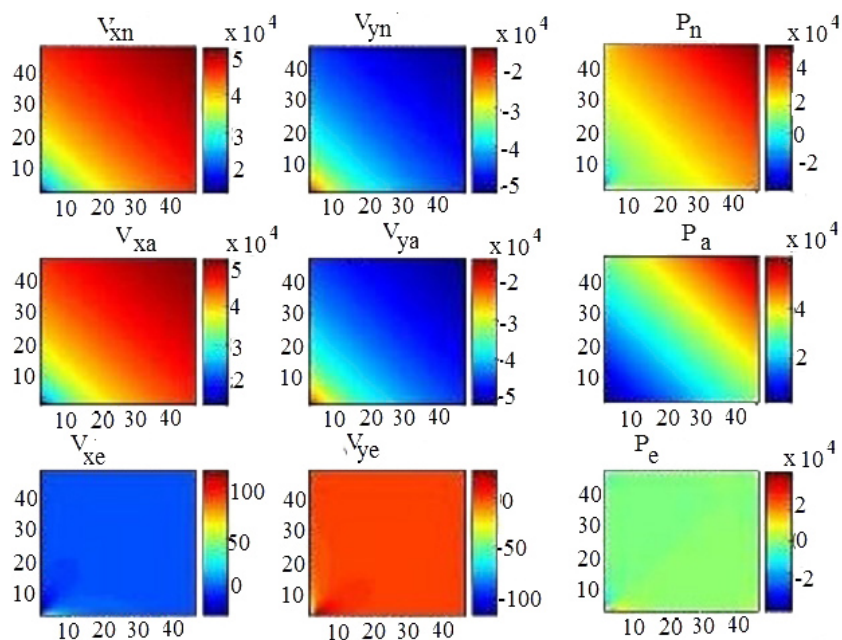

Figure 2. Distribution of $v_{x}, v_{y}$ and $P$; 2-D case, linearly varying viscosity, high-viscosity contrast $\left(\eta_{2}=\eta_{3}=100\right), v_{x e}=v_{x n}-v_{x a}$, $v_{y e}=v_{y n}-v_{y a}, P_{r e}=P_{r n}-P_{r a}$.

Then, due to the uniqueness theorem, the solution of the boundary problem in the domain should coincide with our analytical solution. Let us compute the solution of the boundary problem by a numerical method which is tested. Comparison of the result with the exact analytical solution gives us the error of the numerical solution and shows the quality of the numerical algorithm. In the present paper, we used standard 2-D and 3-D stress-conservative finite-differences on staggered regularly spaced grid for obtaining numerical solutions (Gerya, 2010). Respective MatLab programs for the 2-D and 3-D cases are provided in the Supplement to this paper. All results of calculations are presented in the Supplement. In the main text of the paper we show only a few examples. 


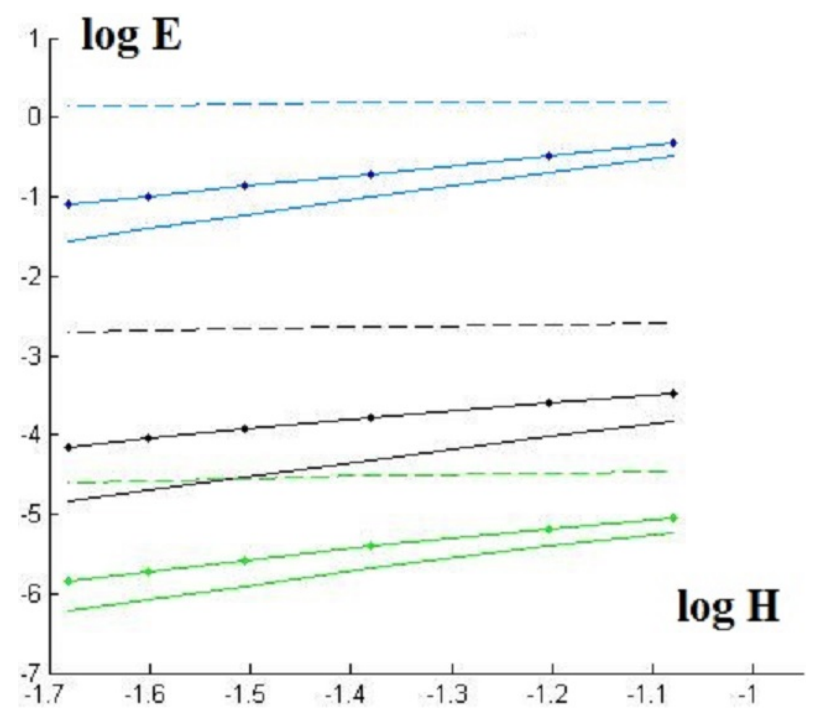

Figure 3. Logarithm of the relative error $(\log E)$ via $\log$ arithm of the grid step $(\log H)$; 2-D case, linearly varying viscosity, highviscosity contrast $\left(\eta_{2}=\eta_{3}=100\right)$; blue lines - pressure, green lines $-v_{x}$, black lines $-v_{y}$; solid lines $-L_{1}$-error, dashed lines $-L_{\infty}$-error, solid dotted line $-L_{2}$-error.

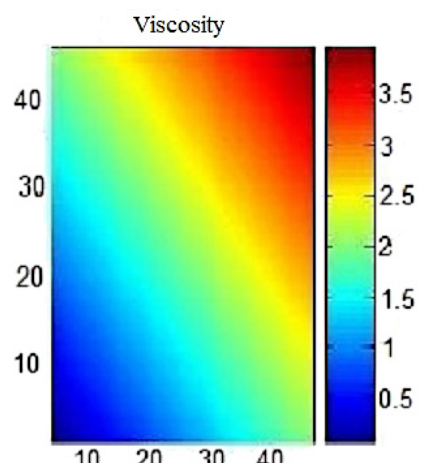

$10 \quad 20 \quad 30 \quad 40$

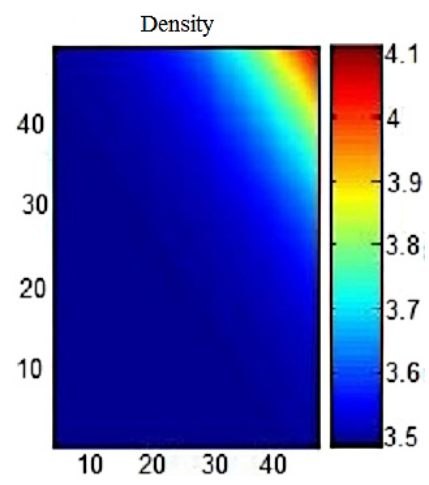

Figure 4. Distribution of viscosity $\eta$ and density $\rho$; 2-D case, exponentially varying viscosity, high-viscosity contrast $\left(\eta_{2}=\eta_{3}=\right.$ $100)$.

\subsection{2-D example}

\subsubsection{Linearly varying viscosity}

Consider a simple example of such flow in a rectangle $\Omega$ : $0 \leq x \leq x_{\text {size }}, 0 \leq y \leq y_{\text {size }}$. We assume that $\eta=a x+b y+c$. We will mark the exact solution obtained in Sect. 2 as $v_{x, a}, v_{y, a}, P_{a}$. It is the solution of the boundary problem in the rectangle $\Omega$ with the following conditions at the boundary $\partial \Omega=\left\{x=0, x=x_{\text {size }}, y=0, y=y_{\text {size }}\right\}$ :

$\left.v_{y}\right|_{\partial \Omega}=v_{y, a},\left.\quad v_{x}\right|_{\partial \Omega}=v_{x, a}$.

Let us compute the velocity and the pressure by the finitedifference scheme. The corresponding solution is marked as $v_{x, n}, v_{y, n}, P_{n}$. The deviation of these values from the exact
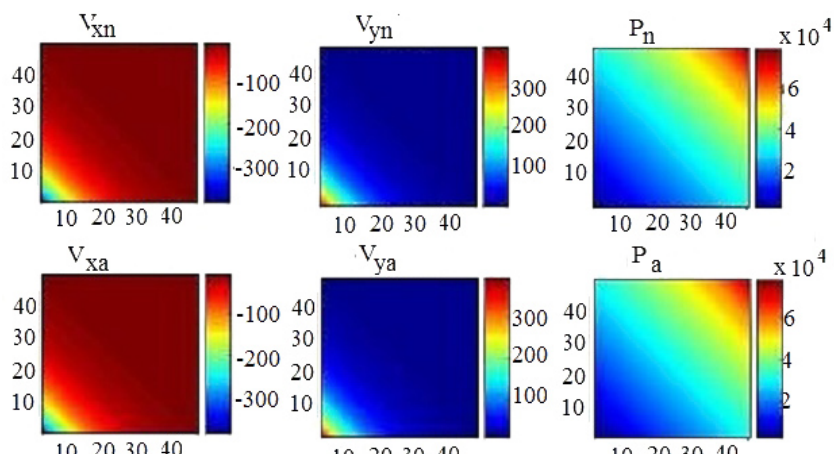

$\mathrm{V}$.

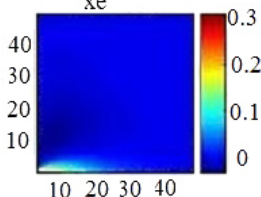

10203040

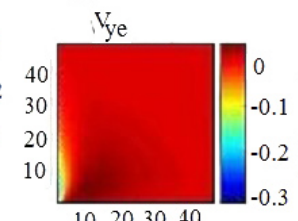

$\mathrm{P}_{\mathrm{e}}$

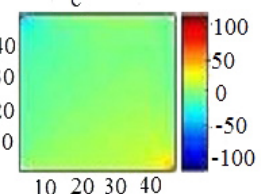

Figure 5. Distribution of $v_{x}, v_{y}$ and $P$; 2-D case, exponentially varying viscosity, high-viscosity contrast $\left(\eta_{2}=\eta_{3}=100\right) v_{x e}=$ $v_{x n}-v_{x a}, v_{y e}=v_{y n}-v_{y a}, P_{r e}=P_{r n}-P_{r a}$.

solution $\left(v_{x, n}-v_{x, a}, v_{y, n}-v_{y, a}, P_{n}-P_{a}\right)$ is related to the error of the numerical scheme. We calculate the relative error norms of three types: $L_{\infty}, L_{1}, L_{2}$ for different viscosity contrasts, i.e., different values of the coefficients $a, b$. We test the program Stokes 2-D variable-viscosity 1 from Gerya (2010). Calculations show that the convergence is better for the case of low-viscosity contrast (cf., Supplement). The results for high-viscosity contrast are presented in Figs. 1-3: Fig. 1 shows prescribed viscosity and density distribution, Fig. 2 presents the pressure and the velocity components distributions and Fig. 3 contains the plot of the relative errors via the grid resolutions in logarithmic scale. The viscosity contrast, i.e., the values of the coefficients in the expression for the viscosity, is determined by the given values of the viscosity at three corners of the model rectangle... The value of the viscosity at the initial rectangle corner is 1 , whereas $\eta_{2}$ and $\eta_{3}$ are the prescribed values of the viscosity at the upper-left and lower-right corner, respectively. In all figures " $n$ " means "numerical solution", " $a$ " means "analytical solution" (benchmark).

For the case of linearly varying viscosity we made calculations for the following system parameters:

$x_{\text {size }}=y_{\text {size }}=1, G_{x}=0, G_{y}=10$,

$\eta_{1}=1, \beta_{1}=1, \beta_{2}=3 \times 10^{3}$,

$c=\eta_{1}, a=\left(\eta_{3}-\eta_{1}\right) / x_{\text {size }}, b=\left(\eta_{2}-\eta_{1}\right) / y_{\text {size }}$,

$\rho=\beta_{1}(a x+b y+c)+\beta_{2}$.

One can see that the numerical approach has rather high accuracy. We observe the conventional situation $-L_{\infty}$-error is the largest among the considered errors norms, and $L_{1}$-error and $L_{2}$-error are similar. 


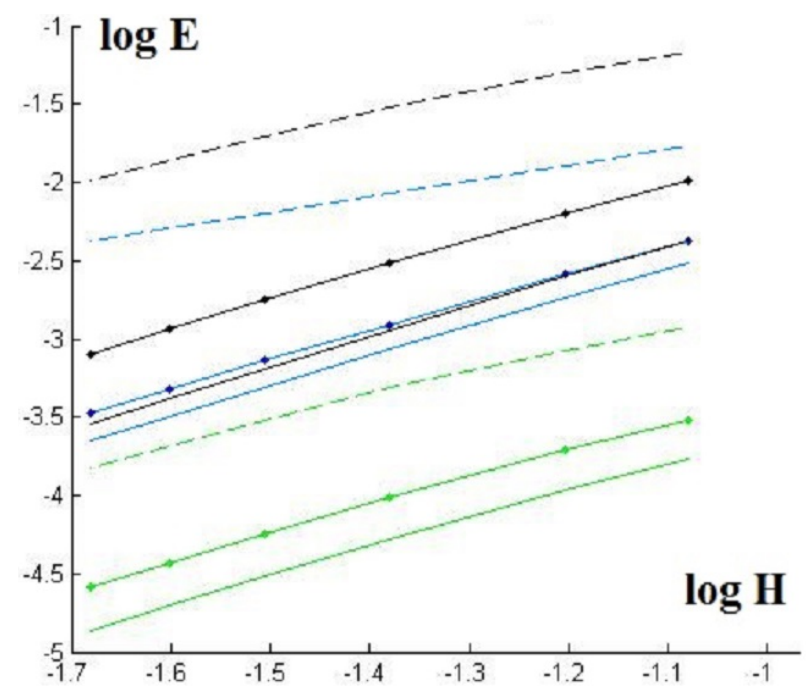

Figure 6. Logarithm of the relative error $(\log E)$ via $\operatorname{logarithm}$ of the grid step $(\log H)$; 2-D case, exponentially varying viscosity, high-viscosity contrast $\left(\eta_{2}=\eta_{3}=100\right)$; blue lines - pressure, green lines $-v_{x}$, black lines $-v_{y}$; solid lines $-L_{1}$-error, dashed lines $-L_{\infty}$-error, solid dotted lines $-L_{2}$-error.
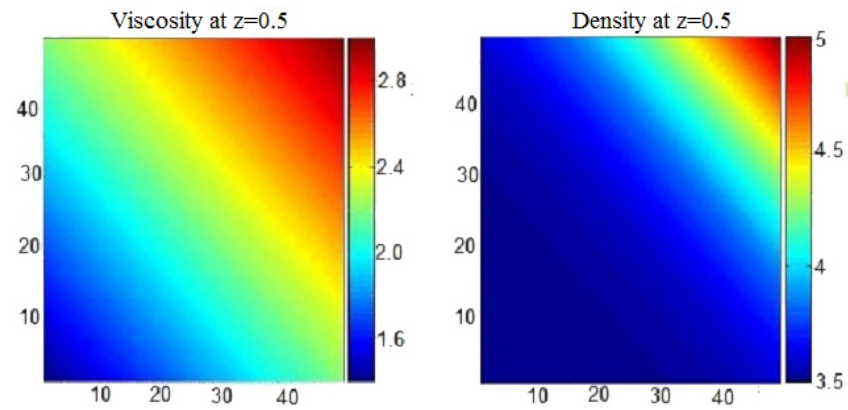

Figure 7. Distribution of viscosity $\eta$ and exponentially varying viscosity, high-viscosity contrast $\left(\eta_{2}=\eta_{3}=\eta_{4}=100\right)$.

To describe in more details the dependence of the error on the viscosity contrast, we fill tables with errors for different values of $\eta_{2}, \eta_{3}$ (see Appendix, Tables A1-A3).

\subsubsection{Exponentially varying viscosity}

The case of exponentially varying viscosity is treated analogously. In order to make a comparison with the case of linearly varying viscosity, we take the same system parameters (geometrical size, gravitational terms and the dependence of the density on the viscosity) with the same viscosity contrast
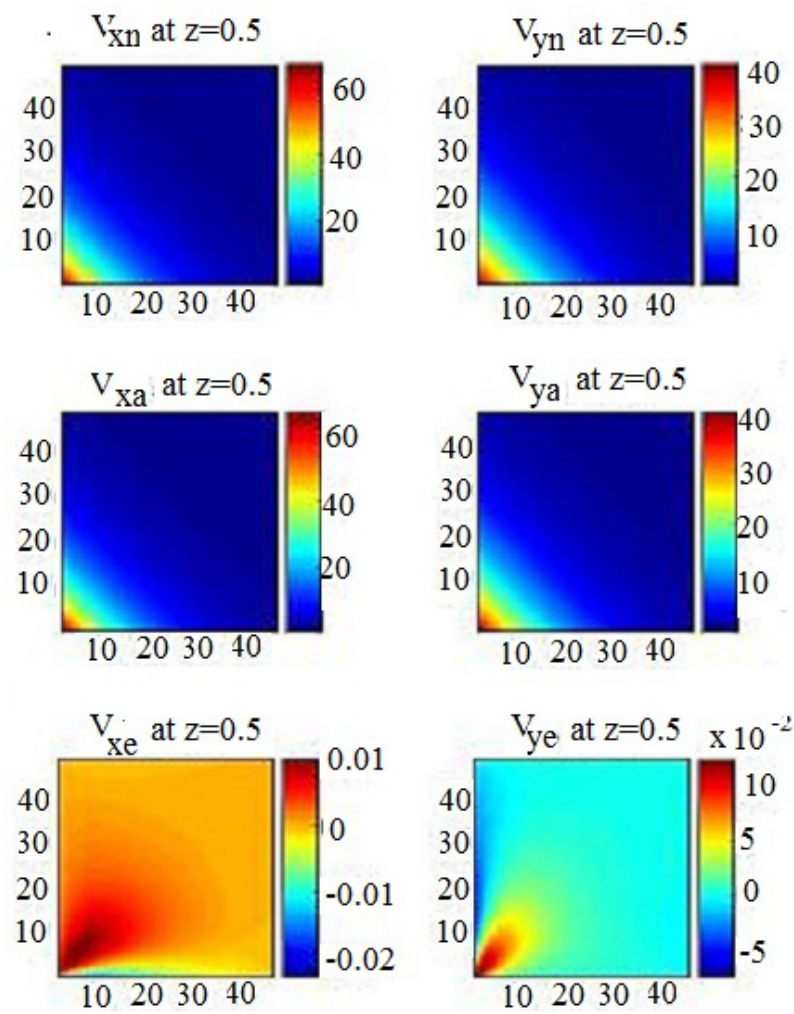

Figure 8. Distribution of $v_{x}$ and $v_{y} ; 3$-D case, exponentially varying viscosity, high-viscosity contrast $\left(\eta_{2}=\eta_{3}=\eta_{4}=100\right) v_{x e}=v_{x n}-$ $v_{x a}, v_{y e}=v_{y n}-v_{y a}$.

(i.e., the values of the viscosity at the rectangle corners):

$C=\eta_{1}, \quad a=\left(\log \left(\eta_{3}\right)-\log \left(\eta_{1}\right)\right) / x_{\text {size }}$,

$b=\left(\log \left(\eta_{2}\right)-\log \left(\eta_{1}\right)\right) / y_{\text {size }}$,

$\eta=C \exp (a x+b y)$,

$\rho=\beta_{1} \eta+\beta_{2}$,

$x_{\text {size }}=y_{\text {size }}=1$,

$G_{x}=10, \quad G_{y}=10$,

$\eta_{1}=1, \quad \beta_{1}=1, \quad \beta_{2}=3 \times 10^{3}$.

We examine two cases (low- and high-viscosity contrasts, cf. Supplement). Figures 4-6 present the results for highviscosity contrast. There are some similarities with the previous case. In particular, $L_{\infty}$ error norm gives us the maximum relative error value among the three considered error norms. However, peculiarities are more interesting. Namely, the numerical scheme works essentially better for the case of exponentially varying viscosity than for linearly varying viscosity. We observe good convergence for both low- and high-viscosity contrasts (compare Figs. 3 and 6, also cf. Supplement).

Dependence of the errors on the viscosity contrast for exponentially varying viscosity is presented in the Appendix Tables A3-A6. 

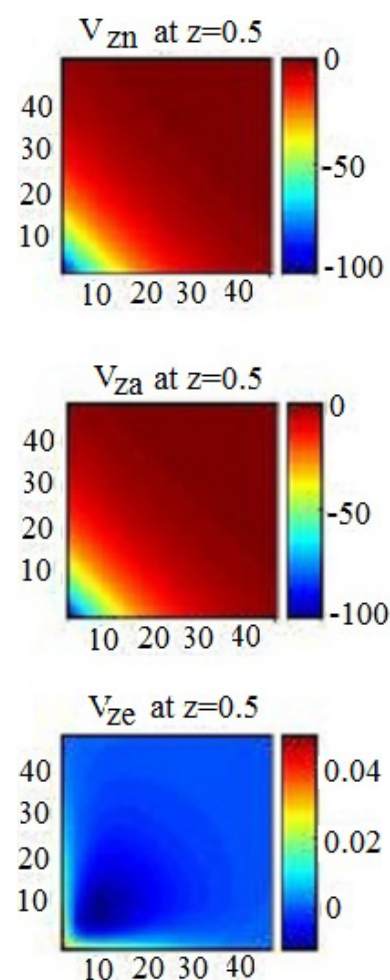
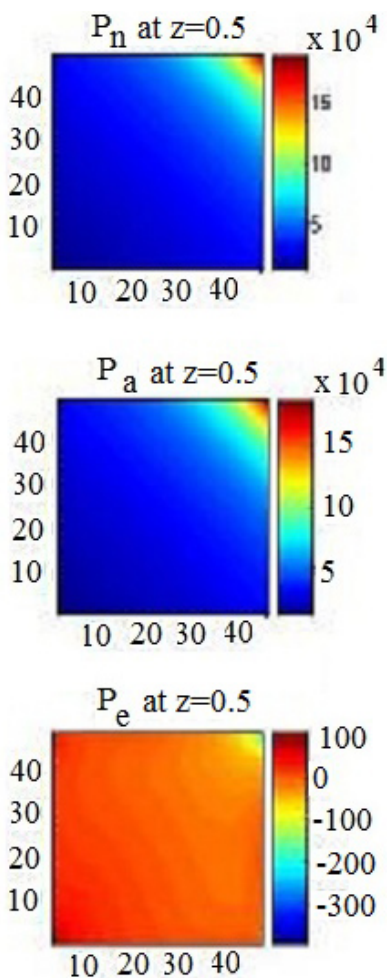

Figure 9. Distribution of $v_{z}$ and $P ; 3-\mathrm{D}$ case, exponentially varying viscosity, high-viscosity contrast $\left(\eta_{2}=\eta_{3}=\eta_{4}=100\right), v_{z e}=$ $v_{z n}-v_{z a}, P_{r e}=P_{r n}-P_{r a}$.

\subsection{3-D example}

One can note that benchmark solutions for the 3-D case are essentially more rare than for the corresponding 2-D situation. It is, therefore, noteworthy that the suggested approach allows us to obtain such solutions for 3-D Stokes and continuity equations. As earlier, we consider the cases of linearly and exponentially varying viscosity.

\subsubsection{Linearly varying viscosity}

The statement of the problem is analogous to the previous case. In the 3-D space we consider a parallelepiped $\Omega: 0 \leq x \leq x_{\text {size }}, 0 \leq y \leq y_{\text {size }}, 0 \leq z \leq z_{\text {size }}$. We assume that $\eta=a x+b y+c z+e$. We will mark the exact solution obtained in Sect. 3 as $v_{x, a}, v_{y, a}, v_{z, a}, P_{a}$. Due to the uniqueness theorem, it is the solution of the boundary problem in the parallelepiped $\Omega$ with the following conditions at the boundary $\partial \Omega=\left\{x=0, x=x_{\text {size }}, y=0, y=y_{\text {size }}, z=0, z=z_{\text {size }}\right\}$ :

$\left.v_{x}\right|_{\partial \Omega}=v_{x, a},\left.v_{y}\right|_{\partial \Omega}=v_{y, a},\left.v_{z}\right|_{\partial \Omega}=v_{z, a}$.

Let us compute the velocity and the pressure numerically using chosen finite-difference algorithm. The corresponding numerical solution is marked as $v_{x, n}, v_{y, n}, v_{z, n}, P_{n}$. The deviation of these values from the exact solution $\left(v_{x, n}-\right.$ $\left.v_{x, a}, v_{y, n}-v_{y, a}, v_{z, n}-v_{z, a}, P_{n}-P_{a}\right)$ is related to the er-

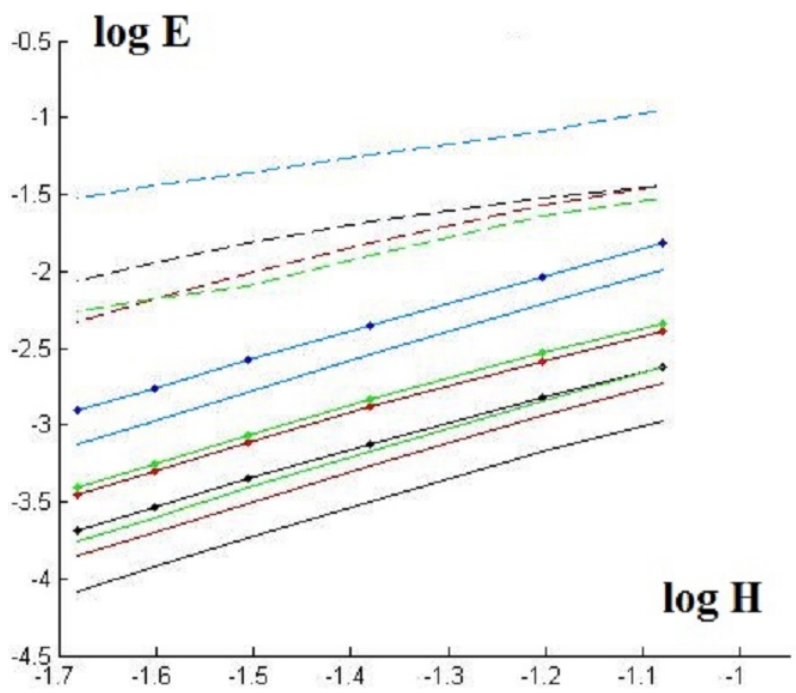

Figure 10. Logarithm of the relative error $(\log E)$ via $\log$ arithm of the grid step $(\log H)$; 3-D case, exponentially varying viscosity, high-viscosity contrast $\left(\eta_{2}=\eta_{3}=\eta_{4}=100\right)$; blue lines - pressure, red lines $-v_{x}$, black lines $-v_{y}$, green lines $-v_{z}$; solid lines $-L_{1^{-}}$ error, dashed lines $-L_{\infty}$-error, solid dotted lines $-L_{2}$-error.

ror of the numerical scheme. As in the 2-D case, we consider three error norms: $L_{\infty}, L_{1}, L_{2}$. We examine cases of low- and high-viscosity contrasts. Coefficients $a, b, c, e$ in the viscosity formula are determined by given viscosity values $\left(\eta_{1}=1, \eta_{2}, \eta_{3}, \eta_{4}\right)$ at four adjacent parallelepiped vertices.

We choose the following system parameters:

$$
\begin{aligned}
& e=\eta_{1}, \quad a=\left(\eta_{3}-\eta_{1}\right) / x_{\text {size }}, \\
& b=\left(\eta_{2}-\eta_{1}\right) / y_{\text {size }}, \quad c=\left(\eta_{4}-\eta_{1}\right) / z_{\text {size }} . \\
& \eta=a x+b y+c z+e, \\
& \rho=\beta_{1} \eta+\beta_{2}, \\
& x_{\text {size }}=y_{\text {size }}=z_{\text {size }}=1, \\
& G_{x}=10, \quad G_{y}=10, \quad G_{z}=0 \\
& \eta_{1}=1, \quad \beta_{1}=1, \quad \beta_{2}=3 \times 10^{3} .
\end{aligned}
$$

Results are presented in the Supplement. It should be mentioned that the numerical procedure for the 3-D case takes essentially greater time than for the 2-D case. Qualitatively, the results are similar to that for the corresponding 2-D case. Better numerical convergence is again found for low-viscosity contrast.

\subsubsection{Exponentially varying viscosity}

As in the 2-D case, we consider also exponentially varying viscosity. The consideration is analogous to that in the respective 2-D case. In order to make a comparison we take here the same system parameters. Naturally, the viscosity and, correspondingly, the density distributions are now exponential. 
The system parameters are chosen as follows:

$$
\begin{aligned}
& C=\eta_{1}, \quad a=\left(\log \left(\eta_{3}\right)-\log \left(\eta_{1}\right)\right) / x_{\text {size }} \\
& b=\left(\log \left(\eta_{2}\right)-\log \left(\eta_{1}\right)\right) / y_{\text {size }} \\
& c=\left(\log \left(\eta_{4}\right)-\log \left(\eta_{1}\right)\right) / z_{\text {size }} \\
& \eta=C \exp (a x+b y+c z) \\
& \rho=\beta_{1} \eta+\beta_{2} \\
& x_{\text {size }}=y_{\text {size }}=z_{\text {size }}=1, \\
& G_{x}=10, \quad G_{y}=10, \quad G_{z}=0 \\
& \eta_{1}=1, \quad \beta_{1}=1, \quad \beta_{2}=3 \times 10^{3}
\end{aligned}
$$

The results are presented in Figs. 7-10 for the case of highviscosity contrast. Similarly to $2-\mathrm{D}$ results, the numerical procedure converges better for the exponentially varying viscosity than for the linearly varying viscosity. The algorithm convergence, i.e., the dependence of the errors on the grid resolution is shown in Fig. 10.

\section{Conclusions}

In this paper, we developed new, specific analytical solutions for the 2-D and 3-D Stokes flows with both linearly and exponentially variable viscosity. We also demonstrated how these solutions can be converted into 2-D and 3-D test problems suitable for benchmarking numerical geodynamic codes. The main advantage of this new generalized approach is that large variety of benchmark problems can be easily generated, including relatively complex cases with open model boundaries, non-vertical gravity and variable gradients of viscosity and density fields, which are not parallel to Cartesian axes. These solutions can be very useful for testing numerical algorithms aimed at modeling variable-viscosity mantle convection and lithospheric dynamics. Examples of respective 2-D and 3-D MatLab codes are provided with the paper.
The Supplement related to this article is available online at doi:10.5194/se-5-461-2014-supplement.

Acknowledgements. The work was made in the framework of Scientific and Technological Cooperation Programme SwitzerlandRussia. This work was partially financially supported by the Government of Russian Federation (grant 074-U01), by the Ministry of Science and Education of the Russian Federation (GOSZADANIE 2014/190, Project 14.Z50.31.0031), by a grant of the Russian Foundation for Basic Research and grants of the President of Russia (state contracts 14.124.13.2045-MK and 14.124.13.1493-MK).

Edited by: J. C. Afonso 
Appendix A: Error decreasing with decreasing grid resolution

The dependence of the convergence on the viscosity contrast is shown in the following tables. Namely, the viscosities $\eta_{2}, \eta_{3}$ run through a set of values from 2 to 10000 . For each pair of $\eta_{2}, \eta_{3}$ we perform the benchmarking procedure described above and obtain the curve describing the dependence of the logarithm of the error norm on the logarithm of the grid resolution. The tangent of the slope angle of this curve gives us an input of the table. Full set of tables are given in the Supplement. Below, we show several examples of such tables.

Table A1. Tangent of the slope of the curve showing the dependence of the logarithm of $L_{2}$-error for pressure $P$ on the logarithm of the grid step for different viscosity contrasts. Linearly varying viscosity.

\begin{tabular}{lrrrrrrr}
\hline \multicolumn{1}{r}{$\eta_{2}$} & 2 & 5 & 20 & 100 & 300 & 1000 & 10000 \\
$\eta_{3}$ & & & & & & & \\
\hline 2 & 1.42 & 1.14 & 1.24 & 0.86 & 0.46 & 0.08 & -0.20 \\
5 & 1.13 & 1.44 & 1.03 & 1.30 & 0.57 & 0.23 & -0.16 \\
20 & 1.23 & 1.03 & 1.39 & 1.27 & 0.83 & 0.43 & -0.01 \\
100 & 1.14 & 1.21 & 1.26 & 1.36 & 1.33 & 0.50 & 0.26 \\
300 & 1.27 & 1.27 & 1.29 & 1.33 & 1.34 & 1.17 & 0.40 \\
1000 & 0.56 & 0.70 & 1.03 & 1.24 & 1.31 & 1.36 & 0.39 \\
10000 & -0.21 & -0.19 & -0.04 & 0.23 & 0.34 & 0.43 & 1.27 \\
\hline
\end{tabular}

Table A2. Tangent of the slope of the curve showing the dependence of the logarithm of $L_{2}$-error for velocity component $v_{x}$ on the logarithm of the grid step for different viscosity contrasts. Linearly varying viscosity.

\begin{tabular}{lrrrrrrr}
\hline \multicolumn{1}{c}{$\eta_{2}$} & 2 & 5 & 20 & 100 & 300 & 1000 & 10000 \\
$\eta_{3}$ & & & & & & & \\
\hline 2 & 1.81 & 1.99 & 1.81 & 1.20 & 0.70 & 0.29 & 0.01 \\
5 & 1.53 & 1.61 & 1.80 & 1.79 & 0.91 & 0.47 & 0.04 \\
20 & 1.47 & 1.54 & 1.61 & 1.81 & 1.09 & 0.77 & 0.20 \\
100 & 1.13 & 1.39 & 1.54 & 1.69 & 2.04 & 0.94 & 0.53 \\
300 & 1.64 & 1.63 & 1.63 & 1.61 & 1.70 & 1.49 & 0.77 \\
1000 & 0.56 & 0.77 & 1.23 & 1.57 & 1.60 & 1.15 & 0.90 \\
10000 & -0.19 & -0.13 & 0.01 & 0.41 & 0.69 & 0.96 & 0.88 \\
\hline
\end{tabular}

Table A3. Tangent of the slope of the curve showing the dependence of the logarithm of $L_{2}$-error for velocity component $v_{y}$ on the logarithm of the grid step for different viscosity contrasts. Linearly varying viscosity.

\begin{tabular}{lrrrrrrr}
\hline \multicolumn{1}{c}{$\eta_{2}$} & 2 & 5 & 20 & 100 & 300 & 1000 & 10000 \\
$\eta_{3}$ & & & & & & & \\
\hline 2 & 1.99 & 1.53 & 1.49 & 1.14 & 0.51 & 0.07 & -0.21 \\
5 & 1.99 & 1.90 & 1.54 & 1.61 & 0.79 & 0.26 & -0.19 \\
20 & 1.81 & 1.80 & 1.79 & 1.56 & 1.24 & 0.63 & -0.03 \\
100 & 1.27 & 1.47 & 1.79 & 2.03 & 1.61 & 0.94 & 0.34 \\
300 & 1.86 & 1.93 & 1.99 & 2.04 & 2.04 & 1.43 & 0.60 \\
1000 & 0.37 & 0.53 & 0.90 & 1.56 & 1.97 & 2.03 & 0.74 \\
10000 & 0.019 & 0.04 & 0.20 & 0.54 & 0.76 & 0.89 & 1.23 \\
\hline
\end{tabular}

\section{A1 Linearly varying viscosity}

Tables A1-A3 contain log rates of $L_{2}$ error norm decreasing with decreasing grid resolution (the curve slope in Fig. 3 in the logarithmic scale) for different viscosity contrasts in the case of linearly varying viscosity. Calculations were made for the following system parameters:

$$
\begin{aligned}
& x_{\text {size }}=y_{\text {size }}=1, \quad G_{x}=0, \quad G_{y}=10, \\
& \eta_{1}=1, \quad \beta_{1}=10^{2}, \quad \beta_{2}=3 \times 10^{3}, \\
& c=\eta_{1}, \quad a=\left(\eta_{3}-\eta_{1}\right) / x_{\text {size }}, \quad b=\left(\eta_{2}-\eta_{1}\right) / y_{\text {size }}, \\
& \rho=\beta_{1}(a x+b y+c)+\beta_{2} .
\end{aligned}
$$




\section{A2 Exponentially varying viscosity}

Tables A4-6 contain log rates of $L_{2}$ error norm decreasing with decreasing grid step (the curve slope in Fig. 6 in the logarithmic scale) for different viscosity contrasts in the case of exponentially varying viscosity. The system parameters are the same as in the case of linearly varying viscosity with respective changes:

$c=\eta_{1}, \quad a=\left(\log \eta_{3}-\log \eta_{1}\right) / x_{\text {size }}$,

$b=\left(\log \eta_{2}-\log \eta_{1}\right) / y_{\text {size }}$,

$\rho=\beta_{1} c \exp (a x+b y)+\beta_{2}$.

Table A4. Tangent of the slope of the curve showing the dependence of the logarithm of $L_{2}$-error for pressure $P$ on the logarithm of the grid step for different viscosity contrasts. Exponentially varying viscosity.

\begin{tabular}{lrrrrrrr}
\hline \multicolumn{1}{c}{$\eta_{2}$} & 2 & 5 & 20 & 100 & 300 & 1000 & 10000 \\
$\eta_{3}$ & & & & & & & \\
\hline 2 & 1.46 & 1.03 & 1.20 & 1.47 & 1.56 & 1.53 & 1.40 \\
5 & 1.03 & 1.13 & 1.23 & 1.39 & 1.53 & 1.51 & 1.39 \\
20 & 1.13 & 1.24 & 1.31 & 1.46 & 1.51 & 1.46 & 1.31 \\
100 & 1.27 & 1.39 & 1.39 & 1.46 & 1.43 & 1.37 & 1.23 \\
300 & 1.37 & 1.41 & 1.39 & 1.40 & 1.37 & 1.31 & 1.19 \\
1000 & 1.41 & 1.41 & 1.36 & 1.33 & 1.30 & 1.24 & 1.11 \\
10000 & 1.37 & 1.34 & 1.26 & 1.17 & 1.14 & 1.11 & 1.00 \\
\hline
\end{tabular}

Table A5. Tangent of the slope of the curve showing the dependence of the logarithm of $L_{2}$-error for velocity component $v_{x}$ on the logarithm of the grid step for different viscosity contrasts. Exponentially varying viscosity.

\begin{tabular}{lrrrrrrr}
\hline \multicolumn{1}{c}{$\eta_{2}$} & 2 & 5 & 20 & 100 & 300 & 1000 & 10000 \\
$\eta_{3}$ & & & & & & & \\
\hline 2 & 1.92 & 2.00 & 1.97 & 2.03 & 2.00 & 1.96 & 1.91 \\
5 & 1.66 & 1.92 & 1.93 & 2.09 & 2.01 & 1.97 & 1.90 \\
20 & 1.63 & 1.84 & 2.06 & 2.09 & 2.01 & 1.96 & 1.87 \\
100 & 1.71 & 1.93 & 2.06 & 2.04 & 1.97 & 1.90 & 1.83 \\
300 & 1.87 & 1.99 & 2.03 & 2.00 & 1.93 & 1.84 & 1.74 \\
1000 & 1.93 & 2.00 & 2.03 & 2.00 & 1.93 & 1.84 & 1.74 \\
10000 & 1.76 & 1.89 & 1.96 & 1.94 & 1.90 & 1.83 & 1.67 \\
\hline
\end{tabular}

Table A6. Tangent of the slope of the curve showing the dependence of the logarithm of $L_{2}$-error for velocity component $v_{y}$ on the logarithm of the grid step for different viscosity contrasts. Exponentially varying viscosity.

\begin{tabular}{lrrrrrrr}
\hline \multicolumn{1}{c}{$\eta_{2}$} & 2 & 5 & 20 & 100 & 300 & 1000 & 10000 \\
$\eta_{3}$ & & & & & & & \\
\hline 2 & 1.97 & 1.66 & 1.81 & 2.10 & 2.01 & 1.94 & 1.83 \\
5 & 2.00 & 1.93 & 1.99 & 2.11 & 2.10 & 2.04 & 1.94 \\
20 & 1.97 & 1.90 & 2.09 & 2.14 & 2.11 & 2.07 & 2.00 \\
100 & 1.89 & 1.96 & 2.11 & 2.09 & 2.06 & 2.04 & 1.97 \\
300 & 2.03 & 2.11 & 2.09 & 2.01 & 1.97 & 1.96 & 1.93 \\
1000 & 2.11 & 2.06 & 2.04 & 1.96 & 1.91 & 1.87 & 1.86 \\
10000 & 1.96 & 1.94 & 1.93 & 1.87 & 1.83 & 1.77 & 1.71 \\
\hline
\end{tabular}




\section{References}

Albers, M.: A local mesh refinement multigrid method for 3-D convection problems with strongly variable viscosity, J. Comput. Phys., 160, 126-150, 2000.

Blankenbach, B., Busse, F., Christensen, U. Cserepes, L., Gunkel, D., Hansen, U., Harder, H., Jarvis, G., Koch, M., Marquart, G., Moore, D., Olson, P., Schmeling H., and Schnaubelt, T.: A benchmark comparison for mantle convection codes, Geophys. J. Int., 98, 23-38, 1989.

Buiter, S. J. H., Babeyko, A. Y., Ellis, S., Gerya, T. V., Kaus, B. J. P., Kellner, A., Schreurs, G., and Yamada, Y.: The numerical sandbox: comparison of model results for a shortening and an extension experiment, in: Analogue and Numerical Modelling of Crustal-Scale Processes, Geol. Soc., London, Special Publications, 253, 29-64, 2006.

Busse, F. H., Christensen, U., Clever, R., Cserepes, L., Gable, C., Giannandrea, E., Guillon, L., Houseman, G., Nataf, H.-C., Ogawa, M., Parmentier, M., Sotin, C., and Travis, B.: 3-D convection at infinite Prandtl number in Cartesian geometry a benchmark comparison, Geophys. Astro. Fluid, 75, 39-59, 1993.

Crameri, F., Schmeling, H., Golabek1, G. J., Duretz, T., Orendt, R., Buiter, S. J. H., May, D. A., Kaus, B. J. P., Gerya, T. V., and Tackley, P. J.: A comparison of numerical surface topography calculations in geodynamic modelling: an evaluation of the "sticky air" method, Geophys. J. Int., 189, 38-54, 2012.

Dabrowski, M., Krotkiewski, M., and Schmid, D. W.: MILAMIN: MATLAB-based finite-element method solver for large problems, Geochem. Geophy. Geosy., 9, Q04030, doi:10.1029/2007GC001719, 2008.

Deubelbeiss, Y. and Kaus, B. J. P.: Comparison of Eulerian and Lagrangian numerical techniques for the Stokes equations in the presence of strongly varying viscosity, Phys. Earth Planet. In., 171, 92-111, 2008.

Duretz, T., May, D. A., Gerya, T. V., and Tackley, P. J.: Discretization errors and free surface stabilization in the finite difference and marker-in-cell method for applied geodynamics: a numerical study, Geochem. Geophy. Geosy., 12, Q07004, doi:10.1029/2011GC003567, 1-26, 2011.

Gerya, T.: Introduction to Numerical Geodynamic Modelling, Cambridge University Press, Cambridge, 2010.

Gerya, T. V. and Yuen, D. A.: Characteristic-based marker-in-cell method with conservative finite-difference schemes for modeling geological flows with strongly variable transport properties, Phys. Earth Planet. In., 140, 293-318, 2003.

Gerya, T. V. and Yuen, D. A.: Robust characteristics method for modeling multiphase visco-elasto-plastic thermo-mechanical problems, Phys. Earth Planet. In., 163, 83-105, 2007.

Gerya, T. V., May, D. A., and Duretz, T.: An adaptive staggered grid finite difference method for modeling geodynamic Stokes flows with strongly variable viscosity, Geochem. Geophy. Geosy., 14, 1200-1225, 2013.

Hager, B. H. and O'Connell, R. J.: A simple global model of plane dynamics and mantle convection, J. Geophys. Res., 86, 48434867, 1981.

Ismail-Zadeh, A. and Tackley, P.: Computational Methods in Geodynamics, Cambridge University Press, Cambridge, 2010.
Kaus, B. J. P.: Factors that control the angle of shear bands in geodynamic numerical models of brittle deformation, Tectonophysics, 484, 36-47, 2010.

Kaus, B. J. P. and Becker, T. W.: Effects of elasticity on the Rayleigh-Taylor instability: implications for large-scale geodynamics, Geophys. J. Int., 168, 843-862, 2007.

Kaus, B. J. P. and Schmalholz, S. M.: 3-D Finite amplitude folding: implications for stress evolution during crustal and lithospheric deformation, Geophys. Res. Lett., 33, L14309, doi:10.1029/2011GC003567, 2006.

Lemiale, V., Muhlhaus, H.-B., Moresi, L. and Stafford, J.: Shear banding analysis of plastic models formulated for incompressible viscous flows, Phys. Earth Planetary Interiors, 171, 177-186, 2008.

Lobanov, I. S., Popov, I. Yu., Popov, A. I., and Gerya, T. V.: Numerical approach to the Stokes problem with high contrasts in viscosity, Appl. Mathemat. Comput., 235, 17-25, 2014.

Moresi, L., Zhong, S., and Gurnis, M.: The accuracy of finite element solutions of Stokes' flow with strongly varying viscosity, Phys. Earth Planet. In., 97, 83-94, 1996.

Moresi, L., Dufour, F., and Mulhaus, H.-B.: A Lagrangian integration point finite element method for large deformation modelingof viscoelastic geomaterials, J. Comput. Phys., 184, 476-497, 2003.

Popov, A. and Sobolev, S.: SLIM3D: a tool for three-dimensional thermomechanical modeling of lithospheric deformation with elasto-visco-plastic rheology, Phys. Earth Planet. In., 171, 5575, 2008.

Popov, I. Yu. and Makeev, I. V.: A benchmark solution for 2-D Stokes flow over cavity, Z. Angew. Math. Phys., 65, 339-348, 2014.

Ramberg, H.: Instability of layered system in the field of gravity, II, Phys. Earth Planet. In., 1, 448-474, 1968.

Revenaugh, J. and Parsons, B.: Dynamic topography and gravity anomalies for fluid layers whose viscosity varies exponentially with depth, Geophys. J. Int., 90, 349-368, 1987.

Schmeling, H., Babeyko, A. Y., Enns, A., Faccenna, C., Funiciello, F., Gerya, T. V., Golabek, G. J., Grigull, S., Kaus, B. J. P., Morra, G., Schmalholz, S. M., and van Hunen, J.: A benchmark comparison of spontaneous subduction models - towards a free surface, Phys. Earth Planet. In., 171, 198-223, 2008.

Schmid, D. V. and Podladchikov, Yu. Yu.: Analytical solutions for deformable elliptical inclusions in general shear, Geophys. J. Int., 155, 269-288, 2003.

Stadler, G., Gurnis, M., Burstedde, C., Wilcox, L. C., Alisic, L., and Ghattas, O.: The dynamics of plate tectonics and mantle flow: from local to global scales, Science, 329, 1033-1038, 2008.

Tackley, P. J.: Modelling compressible mantle convection with large viscosity contrasts in a three-dimensional spherical shell using the yin-yang grid, Phys. Earth Planet. In., 171, 7-18, 2008.

Tackley, P. J. and King, S. D.: Testing the tracer ratio methodfor modeling active compositional fields in mantle convection simulations, Geochem. Geophy. Geosys., 4, 8302, doi:10.1029/2001GC000214, 2003.

Thieulot, C.: FANTOM: two- and three-dimensional numerical modelling of creeping flows for the solution of geological problems, Phys. Earth Plane. Int., 188, 47-68, 2011.

Thieulot, C., Fullsack, P. and Braun, J. Adaptive octree-based finite element analysis of two- and three-dimensional 
indentation problems, J. Geophys. Res., 113, B12207, doi:10.1029/2008JB005591, 2008.

Torrance, K. E. and Turcotte, D. L.: Thermal convection with large viscosity variations, J. Fluid Mech., 47, 113-125, 1971.

Turcotte, D. L. and Schubert, G.: Geodynamics, Cambridge University Press, Cambridge, 2002.

van Keken, P. E., King, S. D., Schmeling, H., Christensen, U. R., Neumeister, D., and Doin, M.-P.: A comparison of methods for the modeling of thermochemical convection, J. Geophys. Res.Sol. Earth., 102, 22477-22495, 1997.

van Keken, P. E., Currie, C., King, S. D., Behn, M. D., Cangleoncle, A., He, J., Katz, R. F., Lin, S.-C., Parmentier, E. M., Spiegelman, M., and Wang, K.: A community benchmark for subduction zone modeling, Phys. Earth Planet. In., 171, 187-197, 2008.
Zhong, S.: Analytic solutions for Stokes flow with lateral variations in viscosity, Geophys. J. Int., 124, 18-28, 1996.

Zhong, S. and Gurnis, M.: The role of plates and temperaturedependent viscosity in phase change dynamics, J. Geophys. Res., 99, 15903-15917, 1994.

Zhong, S., McNamara, A., Tan, E., Moresi, L., and Gurnis, M.: A benchmark study on mantle convection in a 3-D spherical shell using CitcomS, Geochem. Geophy. Geosy., 9, Q10017, doi:10.1029/2008GC002048, 2008. 